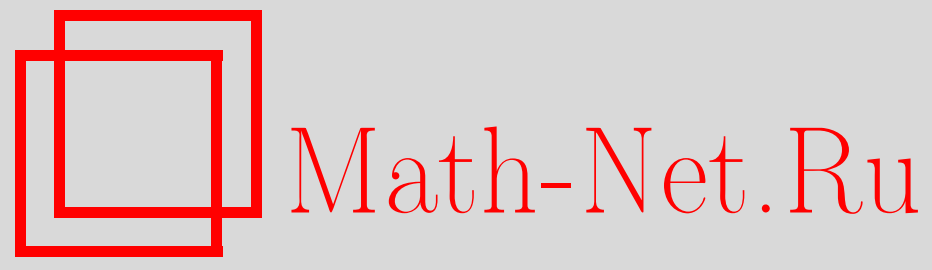

Е. В. Радкевич, K проблеме несуществования диссипативной оценки для дискретных кинетических уравнений, Вестн. Сам. гос. техн. ун-та. Сер. Физ.-мат. науки, 2013, выпуск 1(), 106-143

DOI: https://doi.org/10.14498/vsgtu1140

Использование Общероссийского математического портала Math-Net.Ru подразумевает, что вы прочитали и согласны с пользовательским соглашением

http://www . mathnet.ru/rus/agreement

Параметры загрузки:

IP : 54.224 .135 .184

26 апреля 2023 г., 12:28:01

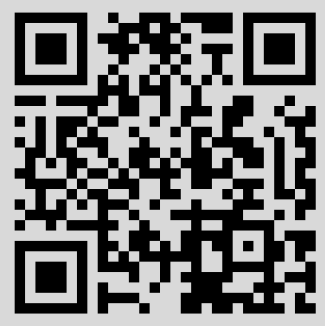


Вестн. Сам. гос. техн. ун-та. Сер. Физ.-мат. науки. 2013. № 1 (30). С. 106-143

УДК 517.958:533.723

\section{К ПРОБЛЕМЕ НЕСУЩЕСТВОВАНИЯ ДИССИПАТИВНОЙ ОЦЕНКИ ДЛЯ ДИСКРЕТНЫХ КИНЕТИЧЕСКИХ УРАВНЕНИЙ}

\section{Е. В. Раджевич}

Московский государственный университет им. М. В. Ломоносова, механико-математический факультет,

Россия, 119899, Москва, Воробьёвы горы.

E-mail: evrad07@gmail.com

Для дискретных уравнений кинетики доказано существование глобального решения в пространствах Соболева, получено разложение его по суммируемости, исследовано влияние осиилляиий, порождаемых оператором взаимодействия. До-

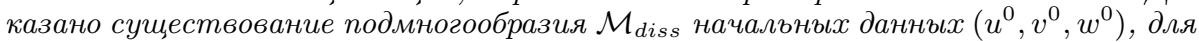
которых существует диссипативное решение. Показано, что при отклонении начальных данных $\left(u^{0}, v^{0}, w^{0}\right)$ от подмногообразия $\mathcal{M}_{\text {diss }}$ оператор взаимодействия порождает недиссипативную часть решения-солитонь (бегущие волнъ). Амплитуда солитонов пропорционалъна расстоянию от $\left(u^{0}, v^{0}, w^{0}\right)$ до подмногообразия $\mathcal{M}_{\text {diss }}$. Отсюда следует стабилизачия решений при $t \rightarrow \infty$ толъко на любом компакте пространственных переменных.

Ключевые слова: диссипативные оценки, дискретнье кинетические уравнения.

1. Введение. В этой статье мы продолжим исследование [1] проблемы несуществования диссипативной оценки Коши для дискретных кинетических уравнений (модели типа Бродуэлла [2] газовой динамики с конечным числом различных скоростей частиц и конечным числом разных парных взаимодействий):

$$
\begin{aligned}
\partial_{t} n_{i}+\left(\omega_{i x} \partial_{x}+\omega_{i y} \partial_{y}\right. & \left.+\omega_{i z} \partial_{z}\right) n_{i}= \\
& =\sum_{k, l, j ; k \neq i, l \neq i, j \neq i} \sigma_{k l}^{i j}\left(n_{k} n_{l}-n_{i} n_{j}\right), \quad i=1,2, \ldots, N,
\end{aligned}
$$

поставленной в 1974 г. в [3]. Общим для таких систем с кинетическим уравнением Больцмана является выполнение уравнения неразрывности

$$
\partial_{t}\left(\sum_{j=1}^{N} n_{j}\right)+\partial_{x} \sum_{j=1}^{N}\left(\omega_{j x} \partial_{x}+\omega_{j y} \partial_{y}+\omega_{j z} \partial_{z}\right) n_{j}=0,
$$

сохранение импульса и справедливость $H$-теоремы Больцмана [4-6]

$$
\begin{aligned}
\partial_{t}\left(n_{i} \ln n_{i}\right)+\left(\omega_{i x} \partial_{x}+\omega_{i y} \partial_{y}+\omega_{i z} \partial_{z}\right)\left(n_{i} \ln n_{i}\right)= \\
\quad=\sum_{k, l, j ; k \neq i, l \neq i, j \neq i} \sigma_{k l}^{i j} \ln \left(\frac{n_{i} n_{j}}{n_{l} n_{k}}\right)\left(n_{k} n_{l}-n_{i} n_{j}\right) .
\end{aligned}
$$

Евгений Владимирович Радкевич (д.ф.-м.н., проф.), профессор, отделение математики, каф. дифференциальных уравнений. 
Отличие состоит в том, что не сохраняется энергия и нет диссипативной оценки. В этой статье мы постараемся выяснить причину отсутствия диссипативной оценки для одномерной модели типа Бродуэлла (см. [3]). Доказательство переносится на двумерную и трёхмерную модели в [3]. Рассмотрим задачу Коши:

$$
\begin{gathered}
\partial_{t} u+\partial_{x} u=\frac{1}{\varepsilon}\left(v^{2}-u w\right), \partial_{t} v=-\frac{2}{\varepsilon}\left(v^{2}-u w\right), \partial_{t} w-\partial_{x} w=\frac{1}{\varepsilon}\left(v^{2}-u w\right) \\
v(0)=v^{0}, \quad u(0)=u^{0}, \quad w(0)=w^{0}
\end{gathered}
$$

на прямой $x \in \mathbb{R}$ для $t>0$. Система (2) является кинетическим уравнением Больцмана модельного одномерного газа [4], состоящего из частиц со скоростями $c=1,0,-1$ (их плотности соответственно $u=n_{1}(x, t), v=n_{2}(x, t)$, $\left.w=n_{3}(x, t)\right)$.

2. Малые возмущения. В окрестности состояния равновесия $v_{e}^{2}=u_{e} w_{e}$ решение будем искать в виде

$$
u=u_{e}+\varepsilon^{2} u_{e}^{1 / 2} \bar{u}, \quad v=v_{e}+\varepsilon^{2} v_{e}^{1 / 2} \bar{v}, \quad w=w_{e}+\varepsilon^{2} w_{e}^{1 / 2} \bar{w} .
$$

Тогда система запишется следующим образом:

$$
\begin{gathered}
\partial_{t} \widehat{U}+A \partial_{x} \widehat{U}+\frac{1}{\varepsilon} B \widehat{U}=\varepsilon \nu^{1 / 2} \Gamma(\widehat{U}, \widehat{U}) \\
\bar{U}(0)=U^{0}
\end{gathered}
$$

где

$$
\begin{gathered}
A=\left(\begin{array}{ccc}
1 & 0 & 0 \\
0 & 0 & 0 \\
0 & 0 & -1
\end{array}\right), \quad B=\left(\begin{array}{ccc}
w_{e} & -2 \sqrt{w_{e} v_{e}} & v_{e} \\
-2 \sqrt{w_{e} v_{e}} & 4 v_{e} & -2 \sqrt{u_{e} v_{e}} \\
v_{e} & -2 \sqrt{u_{e} v_{e}} & u_{e}
\end{array}\right) \\
\nu^{1 / 2}=\left(\begin{array}{c}
w_{e}^{1 / 2} \\
-2 v_{e}^{1 / 2} \\
u_{e}^{1 / 2}
\end{array}\right), \quad \Gamma(U, U)=\widehat{v}^{2}-\widehat{u} \widehat{w}, \quad \widehat{U}=(\widehat{u}, \widehat{v}, \widehat{w})^{\top} .
\end{gathered}
$$

Положим $L_{e}=u_{e}+w_{e}+4 v_{e}$.

ОПреДЕлЕНиЕ. В дальнейшем равномерно стабилизирующееся к нулю решение будем называть диссипативным. Решения, стабилизирующееся на любом компакте, будем называть компактно-диссипативным. K таким решениям можно отнести решения, состоящие из двух частей: диссипативной и суммы солитонов (бегущих волн).

Теорема 1. Пусть начальные данные $\left(u^{0}, v^{0}, w^{0}\right)$ удовлетворяют соотношениям

$$
\begin{gathered}
w_{e}^{1 / 2}\left(\partial_{x}+\frac{1}{\varepsilon} L_{e}\right) w_{0}+\frac{1}{2} v_{e}^{1 / 2} \frac{1}{\varepsilon} L_{e} v_{0}=0, \\
u_{e}^{1 / 2}\left(-\partial_{x}+\frac{1}{\varepsilon} L_{e}\right) u_{0}+\frac{1}{2} v_{e}^{1 / 2} \frac{1}{\varepsilon} L_{e} v_{0}=0 .
\end{gathered}
$$


Тогда существуют постоянные $a_{e, \sigma} u \mu_{0} \in(0,1)$, не зависящие от $\varepsilon$, что для $v^{0} \in H^{2 \sigma}(\mathbb{R}), \sigma>1 / 2$ такого, что норма $\left\|v^{0}\right\|_{H^{2 \sigma}(\mathbb{R})} \leqslant a_{e, \sigma}$, существует глобальное решение задачи Коши для системь $(3),(u, v, w) \in\left(W_{2, \gamma}^{1}\left(\mathbb{R}_{+} ; H^{\sigma}(\mathbb{R})\right)\right)^{3}$, для $\gamma=\mu_{0} \varepsilon$. Здесь норма $f \in H^{\sigma}(\mathbb{R})$ определяется преобразованием Фурье $\widetilde{f}(\xi)$ :

$$
\|f\|_{H^{\sigma}(\mathbb{R})}^{2}=\int_{-\infty}^{\infty}(1+|\xi|)^{2 \sigma}|\tilde{f}(\xi)|^{2} d \xi<\infty
$$

Норма

$$
\|U\|_{W_{2, \gamma}^{1}\left(\mathbb{R}_{+} ;\left(H^{\sigma}(\mathbb{R})\right)^{3}\right)}^{2}=\int_{0}^{\infty} e^{2 \gamma t}\left(\left\|\partial_{t} U\right\|_{\left(H^{\sigma}(\mathbb{R})\right)^{3}}^{2}(t)+\|U\|_{\left(H^{\sigma}(\mathbb{R})\right)^{3}}^{2}(t)\right) d t
$$

\section{Доказательство теоремы 1 мы приведем ниже.}

Множество начальных данных $\left(u^{0}, v^{0}, w^{0}\right)$, для которых существует диссипативное решение, будем называть подмногообразием диссипативности $\mathcal{M}_{\text {diss }}$. Как мы покажем ниже, оператор взаимодействия при отклонении начальных данных $\left(u^{0}, v^{0}, w^{0}\right)$ от подмногообразия $\mathcal{M}_{\text {diss }}$ порождает недиссипативную часть решения - солитоны (бугущие волны). Амплитуда солитонов пропорциональна расстоянию от $\left(u^{0}, v^{0}, w^{0}\right)$ до подмногообразия $\mathcal{M}_{\text {diss }}$. Отсюда следует стабилизация решений при $t \rightarrow \infty$ только на любом компакте (отсутствие диссипативной оценки на всей прямой $x \in \mathbb{R}$ ). Все полученные результаты переносятся на двумерную и трёхмерную модели (1), приведённые в [3].

3. Сведение к одному уравнению. Следуя [1], в образах Фурье по $x$ :

$$
\widehat{U}(t, \xi)=\int_{-\infty}^{\infty} e^{-i \xi x} \widetilde{U}(t, x) d x
$$

систему (3) сводим к системе ОДУ

$$
\begin{aligned}
& u_{e}^{1 / 2}\left(\frac{d}{d t}+i \xi\right) \widetilde{u}(t, \xi)+\frac{1}{\varepsilon}\left(w_{e} u_{e}^{1 / 2} \widetilde{u}(t, \xi)+u_{e} w_{e}^{1 / 2} \widetilde{w}(t, \xi)-2 v_{e} v_{e}^{1 / 2} \widetilde{v}(t, \xi)\right)= \\
& =\varepsilon v_{e} \int_{-\infty}^{\infty}(\widetilde{v}(t, \xi-\eta) \widetilde{v}(t, \eta)-\widetilde{u}(t, \xi-\eta) \widetilde{w}(t, \eta)) d \eta, \\
& w_{e}^{1 / 2}\left(\frac{d}{d t}-i \xi\right) \widetilde{w}(t, \xi)+\frac{1}{\varepsilon}\left(w_{e} u_{e}^{1 / 2} \widetilde{u}(t, \xi)+u_{e} w_{e}^{1 / 2} \widetilde{w}(t, \xi)-2 v_{e} v_{e}^{1 / 2} \widetilde{v}(t, \xi)\right)= \\
& =\varepsilon v_{e} \int_{-\infty}^{\infty}(\widetilde{v}(t, \xi-\eta) \widetilde{v}(t, \eta)-\widetilde{u}(t, \xi-\eta) \widetilde{w}(t, \eta)) d \eta, \\
& -\frac{1}{2} v_{e}^{1 / 2} \frac{d}{d t} \widetilde{v}(t, \xi)+\frac{1}{\varepsilon}\left(w_{e} u_{e}^{1 / 2} \widetilde{u}(t, \xi)+u_{e} w_{e}^{1 / 2} \widetilde{w}(t, \xi)-2 v_{e} v_{e}^{1 / 2} \widetilde{v}(t, \xi)\right)= \\
& =\varepsilon v_{e} \int_{-\infty}^{\infty}(\widetilde{v}(t, \xi-\eta) \widetilde{v}(t, \eta)-\widetilde{u}(t, \xi-\eta) \widetilde{w}(t, \eta)) d \eta \text {. }
\end{aligned}
$$

Как обычно (см. [1]), используя два закона сохранения (законов сохранения для импульса и уравнения неразрывности) для системы (4), понизим 
порядок системы, сведя её к двум уравнениям состояния

$$
\begin{aligned}
& \widetilde{u}(t, \xi)=\frac{1}{u_{e}^{1 / 2}} D^{-}(\xi) e^{-i \xi t}-\frac{1}{2} \frac{v_{e}^{1 / 2}}{u_{e}^{1 / 2}}\left(e^{-\frac{1}{\varepsilon} L_{e} t} v^{0}(\xi)+y(t, \xi)-\right. \\
& \left.\quad-i \xi \int_{0}^{t} e^{-i \xi(t-s)} y(s, \xi) d s+i \xi v^{0}(\xi) \int_{t}^{\infty} e^{-i \xi(t-s)} e^{-\frac{1}{\varepsilon} L_{e} s} d s\right) \\
& \widetilde{w}(t, \xi)=\frac{1}{w_{e}^{1 / 2}} D^{+}(\xi) e^{i \xi t}-\frac{1}{2} \frac{v_{e}^{1 / 2}}{w_{e}^{1 / 2}}\left(e^{-\frac{1}{\varepsilon} L_{e} t} v^{0}(\xi)+y(t, \xi)+\right. \\
& \left.\quad+i \xi \int_{0}^{t} e^{i \xi(t-s)} y(s, \xi) d s-i \xi v^{0}(\xi) \int_{t}^{\infty} e^{i \xi(t-s)} e^{-\frac{1}{\varepsilon} L_{e} s} d s\right)
\end{aligned}
$$

Для

$$
\widetilde{v}(t, \xi)=e^{-\frac{1}{\varepsilon} L_{e} t} \widetilde{v}^{0}(\xi)+y(t, \xi)
$$

и одному уравнению для $y(t, \xi)$ вида

$$
\begin{aligned}
T_{\xi} y(t, \xi)=\frac{1}{\varepsilon} h(t, \xi) & +H(t, \xi)+2 \varepsilon v_{e}^{1 / 2} B(y, y)+2 \varepsilon v_{e}^{1 / 2} L(y)+ \\
+ & \frac{2}{\varepsilon}\left[\frac{w_{e}}{v_{e}^{1 / 2}} D^{-}(\xi) e^{-i \xi t}+\frac{u_{e}}{v_{e}^{1 / 2}} D^{+}(\xi) e^{i \xi t}-\right. \\
& \left.-\varepsilon^{2} \int_{-\infty}^{\infty} D^{-}(\xi-\eta) D^{+}(\eta) e^{-i(\xi-2 \eta) t} d \eta\right],
\end{aligned}
$$

с условием $y(0, \xi)=0$, где

$$
\begin{aligned}
& T_{\xi} y(t, \xi)= \frac{d}{d t} y(t, \xi)+\frac{1}{\varepsilon} L_{e} y(t, \xi)+i \xi \frac{1}{\varepsilon} \int_{0}^{t}\left(u_{e} e^{i \xi(t-s)}-w_{e} e^{-i \xi(t-s)}\right) y(s, \xi) d s \\
& B(y, y)= \int_{-\infty}^{\infty}\{y(t, \xi-\eta) y(t, \eta)- \\
&-\frac{1}{4}\left(y(t, \xi-\eta)-i(\xi-\eta) \int_{0}^{t} e^{-i(\xi-\eta)(t-s)} y(s, \xi-\eta) d s\right) \times \\
&\left.\left.\left.h(t, \xi)=i \xi \widetilde{v}^{0}(\xi) \int_{t}^{\infty}\left(u_{e} e^{i \xi(t-s)}-w_{e} e^{-i \xi(t-s)}\right) e^{-\frac{1}{\varepsilon} L_{e} t} d s, \eta\right)+i \eta \int_{0}^{t} e^{i \eta(t-s)} y(s, \eta) d s\right)\right\} d \eta \\
& H(t, \xi)= 2 \varepsilon v_{e}^{1 / 2} \int_{-\infty}^{\infty}\left\{e^{-2 \frac{1}{\varepsilon} L_{e} t \widetilde{v}^{0}(\xi-\eta) \widetilde{v}^{0}(\eta)+}\right. \\
&+ \frac{1}{2} D^{-}(\xi-\eta) e^{-i(\xi-\eta) t}\left[e^{-\frac{1}{\varepsilon} L_{e} t} v^{0}(\eta)-i \eta v^{0}(\eta) \int_{t}^{\infty} e^{i \eta(t-s)} e^{-\frac{1}{\varepsilon} L_{e} s} d s\right]+ \\
&+\frac{1}{2} \frac{v_{e}^{1 / 2}}{u_{e}^{1 / 2}}\left[i(\xi-\eta) v^{0}(\xi-\eta) \int_{t}^{\infty} e^{-i(\xi-\eta)(t-s)} e^{-\frac{1}{\varepsilon} L_{e} s} d s+e^{-\frac{1}{\varepsilon} L_{e} t} v^{0}(\xi-\eta)\right] \times
\end{aligned}
$$




$$
\begin{aligned}
& \left.\times\left(\frac{1}{w_{e}^{1 / 2}} D^{+}(\eta) e^{i \eta t}-\frac{1}{2} \frac{v_{e}^{1 / 2}}{w_{e}^{1 / 2}}\left[e^{-\frac{1}{\varepsilon} L_{e} t} v^{0}(\eta)-i \eta v^{0}(\eta) \int_{t}^{\infty} e^{i \eta(t-s)} e^{-\frac{1}{\varepsilon} L_{e} s} d s\right]\right)\right\} d \eta \\
& L(y)=\int_{-\infty}^{\infty}\left\{e^{-\frac{1}{\varepsilon} L_{e} t}\left(\widetilde{v}^{0}(\xi-\eta) y(t, \eta)+y(t, \xi-\eta) \widetilde{v}^{0}(\eta)\right)+\right. \\
& +\frac{1}{2} \frac{v_{e}^{1 / 2}}{w_{e}^{1 / 2}}\left(\frac{1}{u_{e}^{1 / 2}} D^{-}(\xi-\eta) e^{-i(\xi-\eta) t}-\frac{1}{2} \frac{v_{e}^{1 / 2}}{u_{e}^{1 / 2}} \times\right. \\
& \left.\times\left[i(\xi-\eta) v^{0}(\xi-\eta) \int_{t}^{\infty} e^{-i(\xi-\eta)(t-s)} e^{-\frac{1}{\varepsilon} L_{e} s} d s+e^{-\frac{1}{\varepsilon} L_{e} t} v^{0}(\xi-\eta)\right]\right) \times \\
& \times\left(y(t, \eta)+i \eta \int_{0}^{t} e^{i \eta(t-s)} y(s, \eta) d s\right)+ \\
& +\frac{1}{2} \frac{v_{e}^{1 / 2}}{u_{e}^{1 / 2}}\left(y(t, \xi-\eta)-i \xi \int_{0}^{t} e^{-i(\xi-\eta)(t-s)} y(s, \xi-\eta) d s\right) \times \\
& \left.\times\left(\frac{1}{w_{e}^{1 / 2}} D^{+}(\eta) e^{i \eta t}-\frac{1}{2} \frac{v_{e}^{1 / 2}}{w_{e}^{1 / 2}}\left[e^{-\frac{1}{\varepsilon} L_{e} t} v^{0}(\eta)-i \eta v^{0}(\eta) \int_{t}^{\infty} e^{i \eta(t-s)} e^{-\frac{1}{\varepsilon} L_{e} s} d s\right]\right)\right\} d \eta \text {. }
\end{aligned}
$$

Здесь

$$
\begin{aligned}
& D^{-}(\xi)=u_{e}^{1 / 2} d^{-}(\xi)+i \xi \frac{1}{2} v_{e}^{1 / 2} v^{0}(\xi) \int_{0}^{\infty} e^{i \xi s} e^{-\frac{1}{\varepsilon} L_{e} s} d s \\
& D^{+}(\xi)=w_{e}^{1 / 2} d^{+}(\xi)-i \xi \frac{1}{2} v_{e}^{1 / 2} v^{0}(\xi) \int_{0}^{\infty} e^{-i \xi s} e^{-\frac{1}{\varepsilon} L_{e} s} d s
\end{aligned}
$$

где

$$
d^{-}(\xi)=u^{0}(\xi)+\frac{1}{2} \frac{v_{e}^{1 / 2}}{u_{e}^{1 / 2}} v^{0}(\xi), \quad d^{+}(\xi)=w^{0}(\xi)+\frac{1}{2} \frac{v_{e}^{1 / 2}}{w_{e}^{1 / 2}} v^{0}(\xi)
$$

По аналогии с [1] мы рассмотрим три типа глобальных решений.

1) Диссипативные решения выделим условием на начальные данные

$$
D^{-}(\xi)=D^{+}(\xi)=0, \quad \forall \xi \in \mathbb{R}^{1} .
$$

Как мы покажем ниже, здесь в согласии с приведённым выше определением решение равномерно стабилизируется к нулю. Тогда уравнение для функции $y(t, \xi)$ :

$$
T_{\xi} y(t, \xi)=\frac{1}{\varepsilon} h(\xi)+H(t, \xi)+2 \varepsilon v_{e}^{1 / 2} B(y, y)+2 \varepsilon v_{e}^{1 / 2} L(y)+y(0, \xi)=0,
$$

те же $B(y, y), h(t, \xi), H(t, \xi)$ и $L(y)$. Здесь, так же как в [1], решение $y(t, \xi)$ будем искать в виде

$$
y(t, \xi)=T_{\xi}^{-1}(Y(t, \xi)) .
$$

Таким образом, неизвестной будет функция $Y(t, \xi) \in L_{2, \gamma}\left(\mathbb{R}_{+}\right)$весового гильбертового пространства с нормой

$$
\|Y\|_{L_{2, \gamma}\left(\mathbb{R}_{+}\right)}=\int_{0}^{\infty} e^{2 \gamma t}|Y(t, \xi)|^{2} d t
$$


Существование и свойства обратного оператора $T_{\xi}^{-1}$ мы исследуем ниже, в п. «Интегро-дифференциальные операторы с трансляцией».

2) Исследование проблемы существования глобального решения задачи Коши для возмущений (3) абсолютно равновесного состояния (см. [1]) показало, что для комплекснозначных начальных данных трудности связаны с появлением секулярного члена

$$
S(t, \xi)=-2 \varepsilon \int_{-\infty}^{\infty} D^{-}(\xi-\eta) D^{+}(\eta) e^{-i(\xi-2 \eta) t} d \eta
$$

в (5), порождаемого оператором взаимодействия. В $x$-представлении получим

$$
\mathcal{S}(t, x)=-2 \varepsilon \mathcal{D}^{-}(x-t) \mathcal{D}^{+}(x+t),
$$

где $\widetilde{\mathcal{S}}(t, \xi)=S(t, \xi), \widetilde{\mathcal{D}^{ \pm}}(\xi)=D^{ \pm}(\xi)$ - преобразования Фурье по $x$. Таким образом, мы имеем мультипликативный эффект оператора взаимодействия, в отличие от аддитивного эффекта оператора взаимодействия для комплексификации

$$
\begin{aligned}
& \partial_{t} \widehat{U}+A \partial_{x} \widehat{U}+\frac{1}{\varepsilon} B \widehat{U}=\varepsilon \nu^{\frac{1}{2}} \operatorname{Re} \Gamma(\widehat{U}, \widehat{U}), \\
& \bar{U}(0)=U^{0}
\end{aligned}
$$

системы (3). Переход к комплексификации (7), как показано в [1], упрощает (6):

$$
S_{M}(t, \xi)=\varepsilon e^{-i \xi t} \int_{-\infty}^{\infty} D^{-}(\xi-\eta) \overline{D^{+}(\eta)} d \eta+\varepsilon e^{i \xi t} \int_{-\infty}^{\infty} \overline{D^{-}(\xi-\eta)} D^{+}(\eta) d \eta
$$

в $x$-представлении -

$$
\mathcal{S}_{M}(t, x)=-\varepsilon\left(\mathcal{K}^{-}(x-t)+\mathcal{K}^{+}(x+t)\right)
$$

Здесь

$$
\begin{gathered}
\widetilde{\mathcal{S}_{M}}(t, \xi)=S_{M}(t, \xi), \quad \widetilde{\mathcal{K}^{-}}(t, \xi)=\int_{-\infty}^{\infty} D^{-}(\xi-\eta) \overline{D^{+}}(\eta) d \eta, \\
\widetilde{\mathcal{K}^{+}}(t, \xi)=\int_{-\infty}^{\infty} \overline{D^{-}}(\xi-\eta) D^{+}(\eta) d \eta
\end{gathered}
$$

- преобразования Фурье по $x$. Существование глобального вещественнозначного решения задачи Коши для системы (3) (см. [1]) следует из существования глобального вещественнозначного решение задачи Коши для комплексификации, что позволяет доказать [1] следующий результат.

Теорема 2. Пусть вещественнозначные начальные данные $\left(u^{0}, v^{0}, w^{0}\right) \in$ $H^{3 \sigma}(\mathbb{R})$ для $\sigma>1 / 2$. Существуют постоянные $k_{e, \sigma}>0, \mu_{0} \in(0,1)$, не зависящие от $\varepsilon$, такие, что при

$$
k_{e, \sigma}\left(\left\|u^{0}\right\|_{H^{3 \sigma}(\mathbb{R})}+\left\|v^{0}\right\|_{H^{3 \sigma}(\mathbb{R})}+\left\|w^{0}\right\|_{H^{3 \sigma}(\mathbb{R})}\right)<\sqrt{\varepsilon}
$$


существует глобальное решение $(u(t, x), v(t, x), w(t, x))$ системы (3) такое, что

$$
\begin{gathered}
u(t, x)-\frac{1}{u_{e}^{1 / 2}} D^{-}(x-t)+\varepsilon \frac{1}{v_{e}^{1 / 2}} \Phi_{-}(x-t) \in W_{2, \gamma}^{1}\left(\mathbb{R}_{+} ; H^{\sigma}(\mathbb{R})\right), \\
w(t, x)-\frac{1}{w_{e}^{1 / 2}} D^{+}(x+t)+\varepsilon \frac{1}{v_{e}^{1 / 2}} \Phi_{+}(x+t) \in W_{2, \gamma}^{1}\left(\mathbb{R}_{+} ; H^{\sigma}(\mathbb{R})\right), \\
v(t, x) \in W_{2, \gamma}^{1}\left(\mathbb{R}_{+} ; H^{\sigma}(\mathbb{R})\right), \gamma=\mu_{0} \varepsilon,
\end{gathered}
$$

где

$$
\begin{aligned}
\left(\frac{1}{\varepsilon} L_{e}-\partial_{x}\right) D^{-}(x) & =u_{e}^{1 / 2}\left(\frac{1}{\varepsilon} L_{e}-\partial_{x}\right) u^{0}(x)+\frac{1}{2} \frac{1}{\varepsilon} v_{e}^{1 / 2} L_{e} v^{0}(x) \\
\left(\frac{1}{\varepsilon} L_{e}+\partial_{x}\right) D^{+}(x) & =w_{e}^{1 / 2}\left(\frac{1}{\varepsilon} L_{e}+\partial_{x}\right) w^{0}(x)+\frac{1}{2} \frac{1}{\varepsilon} v_{e}^{1 / 2} L_{e} v^{0}(x) \\
\Phi_{+}(x) & =\frac{1}{\varepsilon} G^{+}(x)-\frac{1}{2} \varepsilon^{3} \frac{1}{v_{e}^{3 / 2}} \Phi_{-}(x) \Phi_{+}(x) \\
\Phi_{-}(x) & =\frac{1}{\varepsilon} G^{-}(x)-\frac{1}{2} \varepsilon^{3} \frac{1}{v_{e}^{3 / 2}} \Phi_{-}(x) \Phi_{+}(x) \\
G^{+}(x) & =2 \frac{u_{e}}{v_{e}^{1 / 2}} D^{+}(x)-\varepsilon^{2} D^{-}(x) D^{+}(x) \\
G^{-}(x) & =2 \frac{w_{e}}{v_{e}^{1 / 2}} D^{-}(x)-\varepsilon^{2} D^{-}(x) D^{+}(x)
\end{aligned}
$$

для почти всех $x \in \mathbb{R}$. Функиии $\Phi_{ \pm}$определяются условиями несекулярности для оператора взаимодействия (нелинейной части в (3)).

Здесь, в согласии с приведённым выше определением, мы имеем решение компактно-диссипативного типа, которое состоит из суммы солитонов и диссипативной части. Профиль солитонов восстанавливается уравнениями (8) по начальным данным $\left(u^{0}, v^{0}, w^{0}\right)$. Равенство нулю правых частей в $(8)$ отвечает условиям теоремы 1.

Таким образом, для вещественных начальных данных в целом вклад оператора взаимодействия - аддитивный, порождается появлением солитонов при отклонении начальных данных $\left(u^{0}, v^{0}, w^{0}\right)$ от подмногообразия $\mathcal{M}_{\text {diss }}$ начальных данных, для которых есть диссипативное решение. Амплитуда солитонов пропорциональна расстоянию от $\left(u^{0}, v^{0}, w^{0}\right)$ до подмногообразия $\mathcal{M}_{\text {diss }}$. Отсюда следует стабилизация вещественных решений (3) при $t \rightarrow \infty$ только на любом компакте (отсутствие диссипативной оценки на всей прямой).

3) В общем случае (комплекснозначных начальных данных), как мы отмечали выше, трудности исследования связаны с секулярным членом (6). Здесь справедлив следующий результат.

Теорема 3. Пусть комплекснозначные начальные данные $u^{0}, v^{0}, w^{0} \in$ $H^{1+3 \sigma}(\mathbb{R})$ для $\sigma>1 / 2$. Существуют постоянные $k_{e, \sigma}>0, \mu_{0} \in(0,1)$, не зависящие от $\varepsilon$, такие, что при

$$
k_{e, \sigma}\left(\left\|u^{0}\right\|_{H^{1+3 \sigma}(\mathbb{R})}+\left\|v^{0}\right\|_{H^{1+3 \sigma}(\mathbb{R})}+\left\|w^{0}\right\|_{H^{1+3 \sigma}(\mathbb{R})}\right)<\sqrt{\varepsilon}
$$


существует глобальное решение $(u(t, x), v(t, x), w(t, x))$ системь (3) такое, что

$$
\begin{gathered}
u(t, x)-\mathcal{D}_{u}^{-}(x-t)-\mathcal{H}_{u}(x-t, x+t) \in W_{2, \gamma}^{1}\left(\mathbb{R}_{+} ; H^{\sigma}(\mathbb{R})\right), \\
w(t, x)-\mathcal{D}_{w}^{+}(x+t)-\mathcal{H}_{w}(x-t, x+t) \in W_{2, \gamma}^{1}\left(\mathbb{R}_{+} ; H^{\sigma}(\mathbb{R})\right), \\
v(t, x)-\mathcal{D}_{v}^{+}(x+t)-\mathcal{D}_{v}^{-}(x-t)-\mathcal{H}_{v}(x-t, x+t) \in W_{2, \gamma}^{1}\left(\mathbb{R}_{+} ; H^{\sigma}(\mathbb{R})\right),
\end{gathered}
$$

где $\gamma=\mu_{0} \varepsilon ; \mathcal{D}_{u}^{-}(x), \mathcal{D}_{w}^{+}(x), \mathcal{D}_{v}^{ \pm}(x) \in H^{2 \sigma}(\mathbb{R}) ; \mathcal{H}_{u}(x, y), \mathcal{H}_{w}(x, y), \mathcal{H}_{v}(x, y) \in$ $H^{2 \sigma, 1+2 \sigma}\left(\mathbb{R}^{2}\right) \cup H^{1+2 \sigma, 2 \sigma}\left(\mathbb{R}^{2}\right)$ и функиии $\mathcal{H}_{u}(x-t, x+t), \mathcal{H}_{w}(x-t, x+t)$, $\mathcal{H}_{v}(x-t, x+t)-$ конечные суммы произведения солитонов. Отсюда следует стабилизация решений при $t \rightarrow \infty$ только на любом компакте.

Таким образом, в случае комплекснозначных начальных данных в целом вклад оператора взаимодействия - мультипликативный, порождается появлением солитонов и дисперсионной части.

4. Интегро-дифференциальные операторы с трансляцией. Теперь исследуем интегро-дифференциальный оператор с трансляцией

$$
\begin{aligned}
T \mathcal{Y}(t, x) & =\partial_{t} \mathcal{Y}(t, x)+\frac{1}{\varepsilon} L_{e} \mathcal{Y}(t, x)+ \\
+\partial_{x} & \frac{1}{\varepsilon} \int_{0}^{t}\left[u_{e} \mathcal{Y}(t, x+(t-s))-w_{e} \mathcal{Y}(t, x-(t-s)] d s, \quad x \in \mathbb{R}, t>0\right.
\end{aligned}
$$

где

$$
\mathcal{Y}(t, x)=F_{\xi \rightarrow x}^{-1} y
$$

т.е. $y(t, \xi)$ - преобразование Фурье по $x$ функции $\mathcal{Y}(t, x) \in L_{2, \gamma}\left(\mathbb{R}_{+} ; L_{2}(\mathbb{R})\right)$. В образах Фурье по $x$ имеем

$$
T_{\xi} y(t, \xi)=\frac{d}{d t} y(t, \xi)+\frac{1}{\varepsilon} L_{e} y(t, \xi)+i \xi \frac{1}{\varepsilon} \int_{0}^{t}\left(u_{e} e^{i \xi(t-s)}-w_{e} e^{-i \xi(t-s)}\right) y(s, \xi) d s .
$$

Этот оператор, отметим, является линеаризацией уравнения (5). Чтобы получить оценки решения задачи Коши

$$
T_{\xi} y(t, \xi)=f,\left.\quad y(t, \xi)\right|_{t=0}=0
$$

в Соболевских нормах, приведём сначала широко известные факты, которые мы будем использовать в дальнейшем.

ОПРЕДЕлЕНиЕ 1. Назовём пространством Харди $H_{2}(\operatorname{Re} p>\gamma, H)$ класс вектор-функций $\widetilde{f(p)}$ со значениями в сепарабельном гильбертовом пространстве $H$, голоморфных в полуплоскости $\{p \in C: \operatorname{Re} p>\gamma \geqslant 0\}$, для которых

$$
\sup _{x>\gamma} \int_{-\infty}^{+\infty}\|\widetilde{f(x+i y)}\|_{H}^{2} d y<\infty, \quad p=x+i y
$$

Сформулируем теорему Пэли-Винера для пространств Харди. 
ТЕОРЕМА 3 (ПЭЛИ-ВИНЕРА).

1. Пространство $\mathrm{H}_{2}(\operatorname{Re} p>\gamma, H)$ совпадает с множеством вектор-функичй (преобразований Лапласа), допускающих представление

$$
\widetilde{f(p)}=\frac{1}{\sqrt{2 \pi}} \int_{0}^{\infty} e^{p t} f(t) d t
$$

для $f(t) \in L_{2, \gamma}\left(\mathbb{R}_{+}, H\right), p \in C, \operatorname{Re} p>\gamma \geqslant 0$.

2. Для любой вектор-функиии $\widetilde{f(p)} \in H_{2}(\operatorname{Re} p>\gamma, H)$ существует единственное представление (9), где вектор-функиия $f(t) \in L_{2, \gamma}\left(\mathbb{R}_{+}, H\right)$, причём справедлива формула обращения

$$
f(t)=\frac{1}{\sqrt{2 \pi}} \int_{-\infty}^{\infty} e^{(\gamma+i y) t} \widetilde{f(\gamma+i y)} d y, \quad t \in \mathbb{R}_{+}, \gamma \geqslant 0 .
$$

3. Для вектор-функиий $\widetilde{f(p)} \in H_{2}(\operatorname{Re} p>\gamma, H) u f(t) \in L_{2, \gamma}\left(\mathbb{R}_{+}, H\right)$, связанных соотношением (9), справедливо равенство

$$
\begin{aligned}
\|\widetilde{f}\|_{H_{2}(\operatorname{Re} p>\gamma, H)}^{2} \equiv \sup _{x>\gamma} \int_{-\infty}^{+\infty}\|\widetilde{f(x+i y)}\|_{H}^{2} d y= \\
\quad=\int_{0}^{\infty} e^{-2 \gamma t}\|f(t)\|_{N}^{2} d t \equiv\|f\|_{L_{2}\left(\mathbb{R}_{+} ; H\right)}^{2}
\end{aligned}
$$

Лемма 1. Существуют постоянные $a_{0}>0, \mu_{0} \in(0,1)$ такие, что для $\gamma=\mu_{0} \varepsilon$ и любой функиии $f \in L_{2, \gamma}\left(\mathbb{R}_{+}\right)$существует единственное решение $y(t, \xi) \in L_{2, \gamma}\left(\mathbb{R}_{+}\right), \xi \in \mathbb{R}$, уравнения $T_{\xi} y(t, \xi)=f$ такое, что

$$
\left.y(t, \xi)\right|_{t=0}=0
$$

и равномерно по $\xi \in \mathbb{R}$ :

$$
\left\|\frac{d}{d t} y(t, \xi)\right\|_{L_{2, \gamma}\left(\mathbb{R}_{+}\right)}+\|y(t, \xi)\|_{L_{2, \gamma}\left(\mathbb{R}_{+}\right)} \leqslant a_{0}\|f\|_{L_{2, \gamma}\left(\mathbb{R}_{+}\right)} .
$$

Доказ ательст в о леммы 1 приведено в [1].

Лемма 2. Функиии $T_{\xi}^{-1}\left(e^{ \pm i \xi t}\right) \in W_{2, \gamma}^{1}\left(\mathbb{R}_{+}\right)$и существует $a_{0}>0$ такое, что равномерно по $\xi \in \mathbb{R}$ имеем

$$
\left\|T_{\xi}^{-1}\left(e^{ \pm i \xi t}\right)\right\|_{W_{2, \gamma}^{1}\left(\mathbb{R}_{+}\right)} \leqslant a_{0} .
$$

ЛЕмма 2А. Функиии

$J_{1}=-i \xi \int_{0}^{t} e^{-i \xi(t-s)} T_{\xi}^{-1}\left(e^{i \xi s}\right) d s, \quad J_{2}=i \xi \int_{0}^{t} e^{i \xi(t-s)} T_{\xi}^{-1}\left(e^{-i \xi s}\right) d s \in L_{2, \gamma}\left(\mathbb{R}_{+}\right)$, 
и существует $a_{0}>0$ такое, что равномерно по $\xi \in \mathbb{R}$ имеем

$$
\left\|J_{1}\right\|_{L_{2, \gamma}\left(\mathbb{R}_{+}\right)}+\left\|J_{2}\right\|_{L_{2, \gamma}\left(\mathbb{R}_{+}\right)} \leqslant a_{0}
$$

Например, рассмотрим $J_{1}$. Доказ а тельст в о следует из равенства

$$
-i \xi \int_{0}^{t} e^{-i \xi(t-s)} T_{\xi}^{-1}\left(e^{i \xi s}\right) d s=-\int_{0}^{t} e^{-i \xi(t-s)} \frac{d}{d s} T_{\xi}^{-1}\left(e^{i \xi s}\right) d s+T_{\xi}^{-1}\left(e^{i \xi t}\right)
$$

и результатов лемм 1 и 2, в силу которых правая часть (10) принадлежит $L_{2, \gamma}\left(\mathbb{R}_{+}\right)$.

5. Диссипативное решение. Вернёмся к исследованию диссипативного решения, определяемого уравнением

$$
T_{\xi} y(t, \xi)=\frac{1}{\varepsilon} h(t, \xi)+H(t, \xi)+2 \varepsilon v_{e}^{1 / 2} B(y, y)+2 \varepsilon v_{e}^{1 / 2} L(y)+y(0, \xi)=0 .
$$

Так же как в [1], решение $y(t, \xi)$ ищем в виде

$$
y(t, \xi)=T_{\xi}^{-1}(Y(t, \xi)) .
$$

Тогда

$$
\mathcal{B}(Y, Y)=B\left(T_{\xi}^{-1}(Y(t, \xi)), T_{\xi}^{-1}(Y(t, \xi))\right), \quad \mathcal{L}(Y)=L\left(T_{\xi}^{-1}(Y(t, \xi))\right)
$$

и уравнение $(11)$ сведётся к уравнению для $Y(t, \xi)$ в гильбертовом пространстве $L_{2, \gamma}\left(\mathbb{R}_{+}\right)$:

$$
Y(t, \xi)=\frac{1}{\varepsilon} h(t, \xi)+H(t, \xi)+2 \varepsilon v_{e}^{1 / 2} \mathcal{B}(Y, Y)+2 \varepsilon v_{e}^{1 / 2} \mathcal{L}(Y) .
$$

6. Билинейные формы. Рассмотрим билинейную форму

$$
\begin{aligned}
& B(y, y)= \int_{-\infty}^{\infty}\{y(t, \xi-\eta) y(t, \eta)- \\
&-\frac{1}{4}\left[\left(y(t, \xi-\eta)-i(\xi-\eta) \int_{0}^{t} e^{-i(\xi-\eta)(t-s)} y(s, \xi-\eta) d s\right) \times\right. \\
&\left.\left.\times\left(y(t, \eta)+i \eta \int_{0}^{t} e^{i \eta(t-s)} y(s, \eta) d s\right)\right]\right\} d \eta
\end{aligned}
$$

Заметим, что

$$
y(t, \xi) \pm i \xi \int_{0}^{t} e^{ \pm i \xi(t-s)} y(s, \xi) d s=\int_{0}^{t} e^{ \pm i \xi(t-s)} \frac{d}{d s} y(s, \xi) d s .
$$

Для $y=T_{\xi}^{-1}(Y(t, \xi))$ имеем 


$$
\begin{aligned}
& \mathcal{B}(Y, Y)= B\left(T_{\xi}^{-1}(Y(t, \xi)), T_{\xi}^{-1}(Y(t, \xi))\right)= \\
&=\int_{-\infty}^{\infty}\left\{T_{\xi-\eta}^{-1}(Y(t, \xi-\eta)) T_{\eta}^{-1}(Y(t, \eta))-\frac{1}{4}\left[\left(T_{\xi-\eta}^{-1}(Y(t, \xi-\eta))-\right.\right.\right. \\
&\left.\quad-i(\xi-\eta) \int_{0}^{t} e^{-i(\xi-\eta)(t-s)} T_{\eta}^{-1}(\xi-\eta) Y(s, \xi-\eta) d s\right) \times \\
&\left.\left.\quad \times\left(T_{\eta}^{-1}(Y(t, \eta))+i \eta \int_{0}^{t} e^{i \eta(t-s)} T_{\eta}^{-1} Y(s, \eta) d s\right)\right]\right\} d \eta
\end{aligned}
$$

Будем говорить, что $Y(t, \xi) \in L_{2, \gamma}\left(\mathbb{R}_{+} ; \mathcal{H}^{\sigma}(\mathbb{R})\right)$, если ограничена норма

$$
\|Y(t, \xi)\|_{L_{2, \gamma}\left(\mathbb{R}_{+} ; \mathcal{H}^{\sigma}(\mathbb{R})\right)}^{2}=\int_{0}^{\infty} e^{2 \gamma t} \int_{\infty}^{\infty}\left(1+|\xi|^{2}\right)^{\sigma}|Y(t, \xi)|^{2} d \xi d t .
$$

Тогда для обратного преобразования Фурье по пространственной переменной

$$
\mathcal{Y}(t, x)=F_{\xi \rightarrow x}^{-1}(Y(t, \xi))
$$

имеем $\mathcal{Y}(t, x) \in L_{2, \gamma}\left(\mathbb{R}_{+} ; H^{\sigma}(\mathbb{R})\right)$. Для $F(t, \xi) \in L_{2, \gamma}\left(\mathbb{R}_{+} ; \mathcal{H}^{\sigma}(\mathbb{R})\right)$ положим

$$
A_{\sigma}(F)=\|F\|_{L_{2, \gamma}\left(\mathbb{R}_{+} ; \mathcal{H}^{\sigma}(\mathbb{R})\right)} \cdot
$$

Теперь рассмотрим итерационную последовательность

$$
\begin{aligned}
Y^{(n)}(t, \xi)= & \frac{1}{\varepsilon} h(t, \xi)+H(t, \xi)+ \\
& +2 \varepsilon v_{e}^{1 / 2} \mathcal{B}\left(Y^{(n-1)}, Y^{(n-1)}\right)+2 \varepsilon v_{e}^{1 / 2} \mathcal{L}\left(Y^{(n-1)}\right), \quad n \in \mathbb{N}, \\
Y^{0}=F(t, \xi)= & \frac{1}{\varepsilon} h(t, \xi)+H(t, \xi) .
\end{aligned}
$$

Лемма 3. Пусть $A_{2 \sigma}(F)<\infty$ для $\sigma>1 / 2$, тогда существует постоянная $c_{\sigma}$, не зависящая от $\varepsilon$, такая, что при условии

$$
\varepsilon \frac{a_{0}^{4}}{\mu_{0}} c_{\sigma} A_{2 \sigma}^{2}<1
$$

члены итерационной последовательности (13) удовлетворяют оценке

$$
\left\|Y^{(n)}(t, \xi)\right\|_{L_{2, \gamma}\left(\mathbb{R}_{+}\right)} \leqslant\|F(t, \xi)\|_{L_{2, \gamma}\left(\mathbb{R}_{+}\right)}+\frac{A_{2 \sigma}}{(1+|\xi|)^{2 \sigma}}, \quad \forall \xi \in \mathbb{R}, n \in \mathbb{N},
$$

где постоянная $a_{0}$ определяется леммами $1,2$.

Доказ ательств о леммы 3 также приведено в [1].

7. Нелинейное уравнение (диссипативное решение).

СледствиЕ 1. Из леммы 3 для любого биксированного $\xi \in \mathbb{R}$ следует слабая сходимость в $L_{2, \gamma}\left(\mathbb{R}_{+}\right)$итерационной последовательности к функиии $Y(t, \xi) \in L_{2, \gamma}\left(\mathbb{R}_{+}\right)$, удовлетворяющей оченке

$$
\|Y(t, \xi)\|_{L_{2, \gamma}\left(\mathbb{R}_{+}\right)} \leqslant\|F(t, \xi)\|_{L_{2, \gamma}\left(\mathbb{R}_{+}\right)}+\frac{A_{2 \sigma}}{(1+|\xi|)^{2 \sigma}}, \quad \forall \xi \in \mathbb{R}, n \in \mathbb{N} .
$$


Нетрудно доказать, что функция $Y(t, \xi)$ измерима по $\xi$. Из оценки (14) следует, что функция $Y(t, \xi) \in L_{2, \gamma}\left(\mathbb{R}_{+} ; \mathcal{H}^{\sigma}(\mathbb{R})\right)$ и итерационная последовательность сходится к $Y(t, \xi)$ в $L_{2, \gamma}\left(\mathbb{R}_{+} ; \mathcal{H}^{\sigma}(\mathbb{R})\right)$.

ЛЕмма 4. Пусть выполнены условия леммы 3 и

$$
\varepsilon \frac{c_{\sigma}^{2}}{\mu_{0}} a_{0}^{2}\left(a_{0}^{2} A_{2 \sigma}^{2}+\left\|v^{0}\right\|_{\mathcal{H}^{2 \sigma}(\mathbb{R})}^{2}\right)=q^{2}<1 .
$$

Тогда итераиионная последовательность в $L_{2, \gamma}\left(\mathbb{R}_{+} ; \mathcal{H}^{\sigma}\right)$.

фундаментальна

Дока з атель ст в о леммы 4 приведено в [1].

ТЕОрема СУЩЕСтвовАНИЯ. Пусть выполнены условия лемм 3, 4. Тогда существует решение $Y \in L_{2, \gamma}\left(\mathbb{R}_{+} ; \mathcal{H}^{\sigma}(\mathbb{R})\right)$ уравнения $(12)$, удовлетворяющее оиенке

$$
\|Y\|_{L_{2, \gamma}\left(\mathbb{R}_{+}\right)}(\xi) \leqslant\|F(t, \xi)\|_{L_{2, \gamma}\left(\mathbb{R}_{+}\right)}+\frac{A_{2 \sigma}}{(1+|\xi|)^{2 \sigma}}, \quad \forall \xi \in \mathbb{R} .
$$

Доказательство. Существование глобального решения следует из следующих утверждений:

1) из оценки (14) следует, что слабый предел $Y(t, \xi) \in L_{2, \gamma}\left(\mathbb{R}_{+} ; \mathcal{H}^{\sigma}(\mathbb{R})\right)$ и $\mathcal{B}(Y, Y)(t, \xi) \in L_{2, \gamma}\left(\mathbb{R}_{+} ; \mathcal{H}^{\sigma}(\mathbb{R})\right)$

2) из леммы 4 следует, что $Y^{(n)} \rightarrow Y$ в $L_{2, \gamma}\left(\mathbb{R}_{+} ; \mathcal{H}^{\sigma}(\mathbb{R})\right)$;

3) по аналогии с оценками леммы 4 нетрудно доказать, что

$$
\begin{aligned}
& 4 v_{e} \varepsilon^{2}\left\|\mathcal{B}\left(Y^{(n)}, Y^{(n)}\right)-\mathcal{B}(Y, Y)\right\|_{L_{2, \gamma}\left(\mathbb{R}_{+} ; \mathcal{H}^{\sigma}(\mathbb{R})\right)}^{2} \leqslant \\
& \leqslant \varepsilon \frac{c_{\sigma}^{2} a_{0}^{4}}{\mu_{0}} A_{2 \sigma}^{2}\left\|\left(Y^{(n)}-Y\right)\right\|_{L_{2, \gamma}\left(\mathbb{R}_{+} ; \mathcal{H}^{\sigma}(\mathbb{R})\right)}^{2} \\
& 4 v_{e} \varepsilon^{2}\left\|\mathcal{L}\left(\left(Y^{(n)}-Y\right)\right)\right\|_{L_{2, \gamma}\left(\mathbb{R}_{+} ; \mathcal{H}^{\sigma}(\mathbb{R})\right)}^{2} \leqslant \\
& \leqslant \varepsilon^{2} \frac{c_{\sigma}^{2} a_{0}^{4}}{\mu_{0}}\left\|v^{0}\right\|_{2 \sigma}^{2}\left\|\left(Y^{(n)}-Y\right)\right\|_{L_{2, \gamma}\left(\mathbb{R}_{+} ; \mathcal{H}^{\sigma}(\mathbb{R})\right)}^{2}
\end{aligned}
$$

Отсюда следует, что $Y(t, \xi)$ - решение уравнения $(12)$ в $L_{2, \gamma}\left(\mathbb{R}_{+} ; \mathcal{H}^{\sigma}(\mathbb{R})\right)$.

ТЕорема Единственности. Пусть выполнены условия лемм 3, 4. Тогда в классе функиий $Y \in L_{2, \gamma}\left(\mathbb{R}_{+} ; \mathcal{H}^{\sigma}(\mathbb{R})\right)$, удовлетворяющих оченке $(16)$, решение уравнения (12) единственно.

Доказательство. Пусть есть два решения $Y, Y_{1}$ из этого класса. Тогда по аналогии с оценкой (14) можно получить неравенство

$$
\left\|\left(Y-Y_{1}\right)\right\|_{L_{2, \gamma}\left(\mathbb{R}_{+} ; \mathcal{H}^{\sigma}(\mathbb{R})\right)} \leqslant q\left\|\left(Y-Y_{1}\right)\right\|_{L_{2, \gamma}\left(\mathbb{R}_{+} ; \mathcal{H}^{\sigma}(\mathbb{R})\right)},
$$

откуда следует единственность решения. 
8. Условия разрешимости. Теперь перейдём к оценке $A_{2 \sigma}(F)$. Для этого оценим

$$
\begin{aligned}
H(t, \xi)=2 \varepsilon v_{e}^{1 / 2} & \int_{-\infty}^{\infty}\left\{\widetilde { v } ^ { 0 } ( \xi - \eta ) \widetilde { v } ^ { 0 } ( \eta ) \left(e^{-2 \frac{1}{\varepsilon} L_{e} t}-\right.\right. \\
-\frac{1}{4}\left[e^{-\frac{1}{\varepsilon} L_{e} t}+i(\xi-\right. & \left.\eta) \int_{t}^{\infty} e^{-i(\xi-\eta)(t-s)} e^{-\frac{1}{\varepsilon} L_{e} s} d s\right] \times \\
& \left.\left.\times\left[e^{-\frac{1}{\varepsilon} L_{e} t}-i \eta \int_{t}^{\infty} e^{i \eta(t-s)} e^{-\frac{1}{\varepsilon} L_{e} s} d s\right]\right)\right\} d \eta
\end{aligned}
$$

Имеем

$$
\|H(t, \xi)\|_{L_{2, \gamma}\left(\mathbb{R}_{+} ; \mathcal{H}^{2 \sigma}\right)}^{2} \leqslant \varepsilon^{2} 4 v_{e} \mu_{2 \sigma}\left\|v^{0}\right\|_{\mathcal{H}^{2 \sigma}(\mathbb{R})}^{4} \int_{0}^{\infty} e^{2\left(\gamma-\frac{1}{\varepsilon} L_{e}\right) t} d t .
$$

В то же время

$$
\frac{1}{\varepsilon^{2}}\|h\|_{L_{2, \gamma}\left(\mathbb{R}_{+} ; \mathcal{H}^{2 \sigma}(\mathbb{R})\right)}^{2} \leqslant \frac{u_{e}^{2}+w_{e}^{2}}{2 \varepsilon}\left\|v^{0}\right\|_{\mathcal{H}^{2 \sigma}(\mathbb{R})}^{2} \int_{0}^{\infty} e^{2\left(\gamma-\frac{1}{\varepsilon} L_{e}\right) t} d t .
$$

Отсюда следует существование постоянной $c_{\sigma}>0$, не зависящей от $\varepsilon$, такой, что

$$
A_{2 \sigma}^{2} \leqslant \frac{c_{\sigma}^{2}}{\varepsilon}\left\|v^{0}\right\|_{\mathcal{H}^{2 \sigma(}(\mathbb{R})}^{2}\left(1+\varepsilon^{3}\left\|v^{0}\right\|_{\mathcal{H}^{2 \sigma}(\mathbb{R})}^{2}\right) .
$$

Отсюда следует следующее утверждение.

Теорема 4. Пусть для $\sigma>1 / 2$ имеем

$$
\left\|v^{0}\right\|_{\mathcal{H}^{2 \sigma}(\mathbb{R})}^{2} \frac{c_{\sigma}^{2}}{\mu_{0}} a_{0}^{2}\left(1+a_{0}^{2} c_{\sigma}^{2}\left(1+\varepsilon^{3}\left\|v^{0}\right\|_{\mathcal{H}^{2 \sigma}(\mathbb{R})}^{2}\right)\right)<1 .
$$

Тогда существует решение $Y \in L_{2, \gamma}\left(\mathbb{R}_{+} ; \mathcal{H}^{\sigma}(\mathbb{R})\right)$ уравнения $(12)$, удовлетворяющее оценке (16).

Доказ а тель ст во теоремы следует из результатов теоремы существования и оценки (17).

9. Вещественнозначные начальные данные. Как мы отмечали выше, для вещественнозначных начальных данных трудности исследования секулярных членов (6) преодолеваются переходом к комплексификации (7). Существование глобального вещественнозначного решения задачи Коши для системы (3) (см. [1]) следует из существования глобального вещественнозначного решения комплексификации (7). Здесь применимы приведенные выше рассуждения, если уточнить оценки билинейных форм $B(y, y), L(y)$ в этом случае. Для комплексификации билинейная форма

$$
\begin{aligned}
B(y, y) & =\int_{-\infty}^{\infty}\{y(t, \xi-\eta) \overline{y(t, \eta)}- \\
& -\frac{1}{4} \operatorname{Re}\left[\left(y(t, \xi-\eta)-i(\xi-\eta) \int_{0}^{t} e^{-i(\xi-\eta)(t-s)} y(s, \xi-\eta) d s\right) \times\right.
\end{aligned}
$$




$$
\left.\left.\times \overline{\left(y(t, \eta)+i \eta \int_{0}^{t} e^{i \eta(t-s)} y(s, \eta) d s\right)}\right]\right\} d \eta
$$

для

$$
y=T_{\xi}^{-1}(D(t, \xi))+T_{\xi}^{-1}(Y(t, \xi)),
$$

где $D(t, \xi)=\Phi_{\xi} e^{i \xi t}+\Phi_{-\xi} e^{-i \xi t}, Y(t, \xi) \in L_{2, \gamma}\left(\mathbb{R}_{+}\right)$.

\section{Имеем}

$$
\begin{aligned}
B(y, y)= & B\left(T_{\xi}^{-1}(Y(t, \xi)), T_{\xi}^{-1}(Y(t, \xi))\right)+B\left(T_{\xi}^{-1}(D(t, \xi)), T_{\xi}^{-1}(Y(t, \xi))\right)+ \\
& +B\left(T_{\xi}^{-1}(Y(t, \xi)), T_{\xi}^{-1}(D(t, \xi))\right)+B\left(T_{\xi}^{-1}(D(t, \xi)), T_{\xi}^{-1}(D(t, \xi))\right)
\end{aligned}
$$

где

$$
\begin{aligned}
B\left(T_{\xi}^{-1}(Y(t, \xi)), T_{\xi}^{-1}(Y(t, \xi))\right)=\int_{-\infty}^{\infty}\left\{T_{\xi-\eta}^{-1}(Y(t, \xi-\eta)) \overline{T_{\eta}^{-1}(Y(t, \eta))}-\right. \\
-\frac{1}{4} \operatorname{Re}\left[\left(T_{\xi-\eta}^{-1}(Y(t, \xi-\eta))-i(\xi-\eta) \int_{0}^{t} e^{-i(\xi-\eta)(t-s)} T_{\xi-\eta}^{-1} Y(s, \xi-\eta) d s\right) \times\right. \\
\left.\quad \times \overline{\left.\left(T_{\eta}^{-1}(Y(t, \eta))+i \eta \int_{0}^{t} e^{i \eta(t-s)} T_{\eta}^{-1} Y(s, \eta) d s\right)\right]}\right\} d \eta \in L_{2, \gamma}\left(\mathbb{R}_{+}\right) .
\end{aligned}
$$

Здесь

$$
\begin{aligned}
T_{\xi}^{-1}(Y(t, \xi))-i \xi \int_{0}^{t} e^{-i \xi(t-s)} T_{\xi}^{-1} Y(s, \xi) d s & =\int_{0}^{t} e^{-i \xi(t-s)} \frac{d}{d s} T_{\xi}^{-1} Y(s, \xi) d s \\
T_{\xi}^{-1}(Y(t, \xi))+i \xi \int_{0}^{t} e^{i \xi(t-s)} T_{\xi}^{-1} Y(s, \xi) d s & =\int_{0}^{t} e^{i \xi(t-s)} \frac{d}{d s} T_{\xi}^{-1} Y(s, \xi) d s
\end{aligned}
$$

Проблема в том, что

$$
\int_{0}^{t} e^{i \xi(t-s)} T_{\xi}^{-1} D(s, \xi) d s
$$

не принадлежит $L_{2, \gamma}\left(\mathbb{R}_{+}\right)$. В силу леммы 2

$$
i \xi \int_{0}^{t} e^{i \xi(t-s)} T_{\xi}^{-1} e^{-i \xi s} d s, \quad i \xi \int_{0}^{t} e^{-i \xi(t-s)} T_{\xi}^{-1} e^{i \xi s} d s \in L_{2, \gamma}\left(\mathbb{R}_{+}\right),
$$

в то время как

$$
i \xi \int_{0}^{t} e^{-i \xi(t-s)} T_{\xi}^{-1} e^{-i \xi s} d s=-\varepsilon \frac{1}{w_{e}} e^{-i \xi t}+\varepsilon G(t, \xi),
$$

где

$$
G(t, \xi)=\frac{u_{e}}{w_{e}} i \xi \int_{0}^{t} e^{i \xi(t-s)} T_{\xi}^{-1}\left(e^{-i \xi s}\right) d s+\frac{1}{w_{e}}\left(\frac{d}{d t}+\frac{1}{\varepsilon} L_{e}\right) T_{\xi}^{-1}\left(e^{-i \xi t}\right) \in L_{2, \gamma}\left(\mathbb{R}_{+}\right) .
$$


Используя соотношение (18), мы можем преобразовать билинейные формы $B\left(T_{\xi}^{-1}(D(t, \xi)), T_{\xi}^{-1}(D(t, \xi))\right), B\left(T_{\xi}^{-1}(D(t, \xi)), T_{\xi}^{-1}(Y(t, \xi))\right)$ к виду

$$
\begin{aligned}
& 2 \varepsilon v_{e}^{1 / 2} B\left(T_{\xi}^{-1}(D(t, \xi)), T_{\xi}^{-1}(D(t, \xi))\right)=\mathcal{B}(\Phi, \Phi)- \\
& \quad-\frac{1}{2} \varepsilon^{3} \frac{1}{v_{e}^{3 / 2}}\left(e^{i \xi t} \int_{-\infty}^{\infty} \Phi^{-}(\xi-\eta) \overline{\Phi^{+}(\eta)} d \eta+e^{-i \xi t} \int_{-\infty}^{\infty} \overline{\Phi^{-}(\xi-\eta)} \Phi^{+}(\eta) d \eta\right)
\end{aligned}
$$

$2 \varepsilon v_{e}^{1 / 2}\left(B\left(T_{\xi}^{-1}(D(t, \xi)), T_{\xi}^{-1}(Y(t, \xi))\right)+B\left(T_{\xi}^{-1}(Y(t, \xi)) T_{\xi}^{-1}(D(t, \xi))\right)=\mathcal{B}(Y, \Phi)\right.$, где $\mathcal{B}(\Phi, \Phi) \in L_{2, \gamma}\left(\mathbb{R}_{+}\right)$и $\mathcal{B}(\Phi, Y) \in L_{2, \gamma}\left(\mathbb{R}_{+}\right)$; оценка проводится так же, как в п. 5. «Диссипативное решение».

Тогда уравнение (12) в этом случае запишется в виде

$$
\begin{aligned}
& Y(t, \xi)+D(t, \xi)=\frac{1}{\varepsilon} h(t, \xi)+H(t, \xi)-2 \varepsilon v_{e}^{1 / 2} \mathcal{B}(\Phi, \Phi)- \\
&-\frac{1}{2} \varepsilon^{3} \frac{1}{v_{e}^{3 / 2}}\left(e^{i \xi t} \int_{-\infty}^{\infty} \Phi^{-}(\xi-\eta) \overline{\Phi^{+}(\eta)} d \eta+e^{-i \xi t} \int_{-\infty}^{\infty} \overline{\Phi^{-}(\xi-\eta)} \Phi^{+}(\eta) d \eta\right)+ \\
&+ \frac{1}{\varepsilon}\left(\left[2 \frac{w_{e}}{v_{e}^{1 / 2}} D^{-}(\xi)-\varepsilon^{2} \int_{-\infty}^{\infty} D^{-}(\xi-\eta) \overline{D^{+}(\eta)} d \eta\right] e^{-i \xi t}+\right. \\
&+ {\left[2 \frac{u_{e}}{\left.\left.v_{e}^{1 / 2} D^{+}(\xi)-\varepsilon^{2} \int_{-\infty}^{\infty} \overline{D^{-}(\xi-\eta)} D^{+}(\eta) d \eta\right] e^{i \xi t}\right)+}+2 \varepsilon v_{e}^{1 / 2} \mathcal{B}(Y, Y)+2 \varepsilon v_{e}^{1 / 2}(\mathcal{B}(Y, \Phi)+\mathcal{L}(Y))\right.}
\end{aligned}
$$

Таким образом, у нас возникли секулярные неинтегрируемые члены.

10. Условие несекулярности (вещественнозначный случай). Коэффициенты $\Phi^{ \pm}(\xi)$ найдём из условия отсутствия секулярных членов:

$$
\begin{aligned}
& D(t, \xi)=\frac{1}{\varepsilon}( {\left[2 \frac{w_{e}}{v_{e}^{1 / 2}} D^{-}(\xi)-\varepsilon^{2} \int_{-\infty}^{\infty} D^{-}(\xi-\eta) \overline{D^{+}(\eta)} d \eta\right] e^{-i \xi t}+} \\
&+ {\left.\left[2 \frac{u_{e}}{v_{e}^{1 / 2}} D^{+}(\xi)-\varepsilon^{2} \int_{-\infty}^{\infty} \overline{D^{-}(\xi-\eta)} D^{+}(\eta) d \eta\right] e^{i \xi t}\right)- } \\
&-\frac{1}{2} \varepsilon^{3} \frac{1}{v_{e}^{3 / 2}}\left(e^{i \xi t} \int_{-\infty}^{\infty} \Phi^{-}(\xi-\eta) \overline{\Phi^{+}(\eta)} d \eta+e^{-i \xi t} \int_{-\infty}^{\infty} \overline{\Phi^{-(\xi-\eta)}} \Phi^{+}(\eta) d \eta\right)
\end{aligned}
$$

которое можно переписать как алгебраическую систему

$$
\begin{aligned}
& \Phi^{+}(\xi)=\frac{1}{\varepsilon} G^{+}(\xi)-\frac{1}{2} \varepsilon^{3} \frac{1}{v_{e}^{3 / 2}} \int_{-\infty}^{\infty} \Phi^{-}(\xi-\eta) \overline{\Phi^{+}(\eta)} d \eta \\
& \Phi^{-}(\xi)=\frac{1}{\varepsilon} G^{-}(\xi)-\frac{1}{2} \varepsilon^{3} \frac{1}{v_{e}^{3 / 2}} \int_{-\infty}^{\infty} \overline{\Phi^{-}(\xi-\eta)} \Phi^{+}(\eta) d \eta
\end{aligned}
$$


для всех $\xi \in \mathbb{R}$, где

$$
\begin{aligned}
G^{+}(\xi) & =2 \frac{u_{e}}{v_{e}^{1 / 2}} D^{+}(\xi)-\varepsilon^{2} \int_{-\infty}^{\infty} \overline{D^{-}(\xi-\eta)} D^{+}(\eta) d \eta \\
G^{-}(\xi) & =2 \frac{w_{e}}{v_{e}^{1 / 2}} D^{-}(\xi)-\varepsilon^{2} \int_{-\infty}^{\infty} D^{-}(\xi-\eta) \overline{D^{+}(\eta)} d \eta
\end{aligned}
$$

Нам нужны условия, когда существует единственное решение этой системы, такое, что $\Phi^{+}(\xi) \in \mathcal{H}^{2 \sigma}(\mathbb{R})$.

Лемма 5. Пусть $\left(G^{+}, G^{-}\right)(\xi) \in \mathcal{H}^{3 \sigma}(\mathbb{R}), \sigma>1 / 2 u$

$$
\varepsilon c_{e}\left(\left\|u^{0}\right\|_{\mathcal{H}^{3 \sigma}(\mathbb{R})}+\left\|v^{0}\right\|_{\mathcal{H}^{3 \sigma}(\mathbb{R})}+\left\|w^{0}\right\|_{\mathcal{H}^{3 \sigma}(\mathbb{R})}\right) \leqslant 1
$$

¿əe

$$
c_{e}=\frac{v_{e}^{1 / 2}}{u_{e}}\left(u_{e}^{1 / 2}+v_{e}^{1 / 2}+w_{e}^{1 / 2}\right) \max \left(8 \sqrt{2} \frac{u_{e}^{2}}{v_{e}^{2}}, \varepsilon \mu_{3 \sigma}^{1 / 2}\right) .
$$

Тогда существует решение $\left(\Phi^{+}, \Phi^{-}\right)$системь $(20)$ в $\mathcal{H}^{2 \sigma}(\mathbb{R})$, удовлетворяющее оиенке

$$
\left|\Phi^{ \pm}(\xi)\right| \leqslant \frac{1}{\varepsilon}\left(\left|G^{ \pm}(\xi)\right|+\frac{N_{3 \sigma}(G)}{(1+|\xi|)^{3 \sigma}}\right), \quad \forall \xi \in \mathbb{R}
$$

¿de

$$
N_{3 \sigma}=4 \sqrt{2} \frac{u_{e}}{v_{e}^{1 / 2}}\left(u_{e}^{1 / 2}+v_{e}^{1 / 2}+w_{e}^{1 / 2}\right)\left(\left\|u^{0}\right\|_{\mathcal{H}^{3 \sigma}(\mathbb{R})}+\left\|v^{0}\right\|_{\mathcal{H}^{3 \sigma}(\mathbb{R})}+\left\|w^{0}\right\|_{\mathcal{H}^{3 \sigma}(\mathbb{R})}\right)
$$

Более того, решение единственно в классе $\left(\Phi^{+}, \Phi^{-}\right)(\xi)$, удовлетворяющих оценке (21).

Доказ ательст в о леммы 5 приведено в [1].

Решение условия несекулярности позволяет свести уравнение (19) к уравнению в гильбертовом пространстве

$$
\begin{aligned}
Y(t, \xi)=\frac{1}{\varepsilon} h(t, \xi)+H(t, \xi)+2 \varepsilon v_{e}^{1 / 2} \mathcal{B}(\Phi, \Phi)+ \\
+2 \varepsilon v_{e}^{1 / 2} \mathcal{B}(Y, Y)+2 \varepsilon v_{e}^{1 / 2}(\mathcal{B}(Y, \Phi)+\mathcal{L}(Y))
\end{aligned}
$$

вида (11), разрешимость которого доказана в п. 5. «Диссипативное решение».

11. Общий случай (комплекснозначные начальные данные). Теперь перейдём к случаю комплекснозначных начальных данных, трудности рассмотрения которого связаны с секулярным членом (6). Этот факт приводит к необходимости исследования разрешимости уравнения

$$
T_{\xi} X=F_{\xi \rightarrow x}^{-1} S
$$

для специальных правых частей $F_{\xi \rightarrow x}^{-1} S$, где

$$
S(t, \xi)=-\int_{-\infty}^{\infty} e^{-i(\xi-2 \eta) t} D^{-}(\xi-\eta) D^{+}(\eta) d \eta
$$


и $F_{\xi \rightarrow x}^{-1}$ - обратное преобразование Фурье. Ниже мы докажем существование решения задачи Коши

$$
T_{\xi} Z=\varepsilon S(t, \xi),\left.\quad Z(t, \xi)\right|_{t=0}=0
$$

с точностью до правой части $\varepsilon Q^{+}(\xi) e^{i \xi t}+\varepsilon Q^{-}(\xi) e^{-i \xi t}+\varepsilon$ (диссипат) (с точностью до преобразований Фурье по $x$ бегущих волн и диссипативного члена (экспоненциально быстро убывающего)).

Мы рассмотрим более общую правую часть вида

$$
S_{D}(t, \xi)=\int_{-\infty}^{\infty} e^{-i(\xi-2 \eta) t} D(\xi-\eta, \eta) d \eta
$$

Решением $\left(Z_{D}(t, \xi), Q_{D}^{ \pm}(\xi), F_{D}(t, \xi)\right)$ задачи Коши $(22)$ с точностью до правой части $Q_{D}^{+}(\xi) e^{i \xi t}+Q_{D}^{-}(\xi) e^{-i \xi t}+\varepsilon F_{D}(t, \xi)$ будем называть решение следующей задачи:

$$
T_{\xi}\left(Z_{D}(t, \xi)\right)=\varepsilon S_{D}(t, \xi)+Q_{D}^{+} e^{i \xi t}+Q_{D}^{-} e^{-i \xi t}+\varepsilon F_{D},\left.\quad Z_{D}(t, \xi)\right|_{t=0}=0
$$

где $F_{D} \in L_{2, \gamma}\left(\mathbb{R}_{+}\right), \xi \in \mathbb{R}$. Обратное преобразовании Фурье $F_{\xi \rightarrow x}^{-1}$ имеет следующий вид:

$$
F_{\xi \rightarrow x}^{-1}\left(S_{D}\right)(t, x)=\mathcal{D}(x-t, x+t)
$$

где

$$
F_{x \rightarrow \xi, y \rightarrow \eta}(\mathcal{D})(\xi, \eta)=D(\xi, \eta)
$$

- преобразование Фурье функции $\mathcal{D}(x, y)$. Тогда

$$
\begin{aligned}
& \left\|S_{D}(t, \cdot)\right\|_{\mathcal{H}^{\sigma}(\mathbb{R})}^{2} \leqslant \\
& \leqslant 2\left\{\int_{-\infty}^{\infty} \frac{d \eta}{(1+|\eta|)^{2 \sigma}}\left(\int_{-\infty}^{\infty} \int_{-\infty}^{\infty}(1+|\eta|)^{2 \sigma}(1+|\xi-\eta|)^{2 \sigma}(D(\xi-\eta, \eta))^{2} d \xi d \eta\right)+\right. \\
& \quad+\int_{-\infty}^{\infty} \int_{-\infty}^{\infty}(1+|\eta|)^{2 \sigma}(1+|\xi-\eta|)^{2 \sigma}(D(\xi-\eta, \eta))^{2} d \eta \times \\
& \left.\quad \times\left(\int_{-\infty}^{\infty} \frac{d \eta}{(1+|\xi-\eta|)^{2 \sigma}}\right) d \xi\right\} \leqslant 4 \mu_{\sigma}^{2}\|D\|_{\mathcal{H}^{\sigma, \sigma}\left(\mathbb{R}^{2}\right)}^{2}, \quad \mu_{\sigma}^{2}=\int_{-\infty}^{\infty} \frac{d \eta}{(1+|\eta|)^{2 \sigma}} .
\end{aligned}
$$

Лемма 6. Пусть $D(\xi, \eta) \in \mathcal{H}^{\sigma+1, \sigma}\left(\mathbb{R}^{2}\right) \cap \mathcal{H}^{\sigma, \sigma+1}\left(\mathbb{R}^{2}\right)$. Тогда существует решение $Z_{D}(t, \xi), Q_{D}^{ \pm}(\xi), F_{D}(t, \xi)$ задачи Коши

$$
T_{\xi}\left(Z_{D}(t, \xi)\right)=\varepsilon S_{D}(t, \xi)+Q_{D}^{+} e^{i \xi t}+Q_{D}^{-} e^{-i \xi t}+\varepsilon F_{D}(t, \xi),\left.\quad Z_{D}(t, \xi)\right|_{t=0}=0
$$

maкое, что

$$
\begin{gathered}
Z_{D}(t, \xi)=\varepsilon \int_{-\infty}^{\infty} \int_{0}^{t} e^{-i(\xi-\eta)(t-s)} \Xi_{D}(\xi-\eta, \eta, s) e^{i \eta(t-s)} d s d \eta= \\
=\varepsilon \int_{-\infty}^{\infty} e^{-i(\xi-\eta) t} \Psi_{D}(\xi-\eta, \eta) e^{i \eta t} d \eta+
\end{gathered}
$$




$$
+R_{D}^{+}(\xi) e^{i \xi t}+R_{D}^{-}(\xi) e^{-i \xi t}+\varepsilon G_{D}(t, \xi)
$$

¿əe

$$
\begin{gathered}
\varepsilon\left\|\int_{-\infty}^{\infty} e^{-i(\xi-\eta) t} \Psi_{D}(\xi-\eta, \eta) e^{i \eta t} d \eta\right\|_{W_{\infty}^{1}\left(\mathbb{R}_{+} ; \mathcal{H}^{\sigma}(\mathbb{R})\right.} \leqslant \\
\leqslant c_{0} \varepsilon^{1 / 2}\left(\|D\|_{\mathcal{H}^{\sigma+1, \sigma}\left(\mathbb{R}^{2}\right)}+\|D\|_{\mathcal{H}^{\sigma, \sigma+1}\left(\mathbb{R}^{2}\right)}\right) \\
\left\|e^{ \pm i \xi t} R_{D}^{ \pm}(\xi)\right\|_{W_{\infty}^{1}\left(\mathbb{R}_{+} ; \mathcal{H}^{\sigma}(\mathbb{R})\right.} \leqslant c_{0} \varepsilon^{1 / 2}\left(\|D\|_{\mathcal{H}^{\sigma+1, \sigma}\left(\mathbb{R}^{2}\right)}+\|D\|_{\mathcal{H}^{\sigma, \sigma+1}\left(\mathbb{R}^{2}\right)}\right) \\
\left\|Q_{D}^{+}\right\|_{\mathcal{H}^{\sigma}(\mathbb{R})} \leqslant \varepsilon^{1 / 2} c_{0}\|D\|_{\mathcal{H}^{\sigma, \sigma}\left(\mathbb{R}^{2}\right)}, \quad \varepsilon\left\|F_{D}\right\|_{L_{2, \gamma}\left(\mathbb{R}_{+} ; \mathcal{H}^{\sigma}(\mathbb{R})\right)} \leqslant c_{0}\|D\|_{\mathcal{H}^{\sigma, \sigma}\left(\mathbb{R}^{2}\right)} .
\end{gathered}
$$

Доказательство.

Построение решения $Z_{D}(t, \xi), Q_{D}^{ \pm}(\xi), F_{D}(t, \xi)$.

Для $2 q<1, q>0$, рассмотрим непересекающиеся интервалы

$$
I_{1}(\xi)=\{\eta \in \mathbb{R},|\eta|<q|\xi|\}, \quad I_{2}(\xi)=\{\eta \in \mathbb{R},|\xi-\eta|<q|\xi|\}, \quad I_{0}=\mathbb{R} \backslash\left(I_{1} \cup I_{2}\right) .
$$

Сделав преобразование Лапласа по $t$, положим

$$
\begin{aligned}
\widehat{S_{D}}(p, \xi)=\int_{-\infty}^{\infty} \frac{D(\xi-\eta, \eta)}{p+i(\xi-2 \eta)} d \eta= & \int_{I_{0}} \frac{D(\xi-\eta, \eta)}{p+i(\xi-2 \eta)} d \eta+ \\
& +\int_{I_{1}} \frac{D(\xi-\eta, \eta)}{p+i(\xi-2 \eta)} d \eta+\int_{I_{2}} \frac{D(\xi-\eta, \eta)}{p+i(\xi-2 \eta)} d \eta
\end{aligned}
$$

1) В области $I_{0}$, где $|\eta|>q|\xi|,|\xi-\eta|>q|\xi|$, решим уравнение

$$
\begin{aligned}
& T_{\xi}\left[\int_{I_{0}} \int_{0}^{t} \Xi_{0}(\xi, \eta, s) e^{i(2 \eta-\xi)(t-s)} d s d \eta\right]= \\
& \quad=\varepsilon S_{0}(t, \xi)+Q^{+}(\xi) e^{i \xi t}+Q^{-}(\xi) e^{-i \xi t}+\varepsilon F_{0}(t, \xi), \quad \xi \in I_{0},
\end{aligned}
$$

где

$$
\widehat{S_{0}}(p, \xi)=\int_{I_{0}} \frac{D(\xi-\eta, \eta)}{p+i(\xi-2 \eta)} d \eta,
$$

- преобразование Лапласа $S_{0}$ по $t$ и $F_{0}(t, \xi) \in L_{2, \gamma}\left(\mathbb{R}_{+} ; \mathcal{H}^{\sigma}(\mathbb{R})\right)$.

1a) Сделав преобразование Лапласа по $t$, получим

$$
\begin{aligned}
J_{0} & =\left(p+\frac{1}{\varepsilon} L_{e}+\frac{1}{\varepsilon}\left(\frac{i \xi u_{e}}{p-i \xi}-\frac{i \xi w_{e}}{p+i \xi}\right)\right) \int_{I_{0}} \frac{\widehat{\Xi_{0}}(\xi, \eta, p)}{p+i \xi-2 i \eta} d \eta= \\
& =\left(p+\frac{1}{\varepsilon} L_{e}\right) \int_{I_{0}} \frac{\widehat{\Xi_{0}}(\xi, \eta, p)}{p+i \xi-2 i \eta} d \eta+\frac{1}{\varepsilon} \int_{I_{0}}\left(\frac{i \xi u_{e}}{p-i \xi}-\frac{i \xi w_{e}}{p+i \xi}\right) \frac{\widehat{\Xi}(\xi, \eta, p)}{p+i \xi-2 i \eta} d \eta
\end{aligned}
$$

где для $\eta \neq 0, \xi-\eta \neq 0$ имеем 


$$
\begin{aligned}
&\left(\frac{i \xi u_{e}}{p-i \xi}-\frac{i \xi w_{e}}{p+i \xi}\right) \frac{1}{p+i \xi-2 i \eta}=-\left(\frac{\xi}{2(\xi-\eta)} u_{e}+\frac{\xi}{2 \eta} w_{e}\right) \frac{1}{p+i \xi-2 i \eta}+ \\
&+\frac{\xi}{2(\xi-\eta)} u_{e} \frac{1}{p-i \xi}+\frac{\xi}{2 \eta} w_{e} \frac{1}{p+i \xi}
\end{aligned}
$$

Тогда

$$
\begin{aligned}
J_{0}= & \int_{I_{0}}\left(p+\frac{1}{\varepsilon}\left[L_{e}-\frac{\xi}{2(\xi-\eta)} u_{e}-\frac{\xi}{2 \eta} w_{e}\right]\right) \frac{\widehat{\Xi_{0}}(\xi, \eta, p)}{p+i \xi-2 i \eta} d \eta+ \\
+ & u_{e} \frac{1}{p-i \xi} \int_{I_{0}} \frac{\xi}{2(\xi-\eta)} \widehat{\Xi_{0}}(\xi, \eta, p) d \eta+w_{e} \frac{1}{p+i \xi} \int_{I_{0}} \frac{\xi}{2 \eta} \widehat{\Xi_{0}}(\xi, \eta, p) d \eta= \\
& =\varepsilon \int_{I_{0}} \frac{D(\xi-\eta, \eta)}{p+i(\xi-2 \eta)} d \eta
\end{aligned}
$$

На множестве $\eta \in I_{0}$ положим

$$
\widehat{\Xi_{0}}(\xi, \eta, p)=\varepsilon \frac{D(\xi-\eta, \eta)}{p+\frac{1}{\varepsilon}\left[L_{e}-\frac{\xi}{2(\xi-\eta)} u_{e}-\frac{\xi}{2 \eta} w_{e}\right]}, \quad \eta \in I_{0} .
$$

Отсюда

$$
J_{0}-\varepsilon \int_{I_{0}} \frac{D(\xi-\eta, \eta)}{p+i(\xi-2 \eta)} d \eta=q^{+}+q^{-}
$$

$q^{+}=-u_{e} \frac{1}{p-i \xi} \int_{I_{0}} \frac{\xi}{2(\xi-\eta)} \widehat{\Xi_{0}}(\xi, \eta, p) d \eta, \quad q^{-}=-w_{e} \frac{1}{p+i \xi} \int_{I_{0}} \frac{\xi}{2 \eta} \widehat{\Xi_{0}}(\xi, \eta, p) d \eta$

На интервале $I_{0}$ имеем

$$
L_{e}-\frac{\xi}{2(\xi-\eta)} u_{e}-\frac{\xi}{2 \eta} w_{e} \geqslant L_{e}\left(1-\frac{u_{e}+w_{e}}{2 q L_{e}}\right)=a_{0}>0,
$$

если

$$
1-\frac{u_{e}+w_{e}}{2 q L_{e}}>0 \Longrightarrow 1>2 q>\frac{u_{e}+w_{e}}{L_{e}}=\frac{1}{1+\frac{4 v_{e}}{u_{e}+w_{e}}}
$$

Отсюда

$$
e^{-\frac{1}{\varepsilon}\left[L_{e}-\frac{\xi}{2(\xi-\eta)} u_{e}-\frac{\xi}{2 \eta} w_{e}\right] t} \leqslant e^{-\frac{1}{2} \frac{1}{\varepsilon} a_{0} t}, \quad \forall \eta \in I_{0} .
$$

Сделав обратное преобразование Лапласа $(p \rightarrow t)$, в образах $t$ имеем

$$
\begin{aligned}
& L_{p \rightarrow t} q^{+}=-u_{e} \int_{0}^{t} e^{i \xi(t-s)} \int_{I_{0}} \frac{\xi}{2(\xi-\eta)} \Xi_{0}(\xi, \eta, s) d s d \eta= \\
&=-u_{e} e^{i \xi t} \int_{0}^{\infty} e^{-i \xi} \int_{I_{0}} \frac{\xi}{2(\xi-\eta)} \Xi_{0}(\xi, \eta, s) d s d \eta+ \\
& \quad+u_{e} \int_{t}^{\infty} e^{i \xi(t-s)} \int_{I_{0}} \frac{\xi}{2(\xi-\eta)} \Xi_{0}(\xi, \eta, s) d s d \eta .
\end{aligned}
$$


Также

$$
\begin{aligned}
L_{p \rightarrow t} q^{-}=-w_{e} e^{-i \xi t} \int_{0}^{\infty} e^{i \xi s} \int_{I_{0}} \frac{\xi}{2 \eta} \Xi_{0}(\xi, \eta, s) d s d \eta+ & \\
& +w_{e} \int_{t}^{\infty} e^{-\xi(t-s)} \int_{I_{0}} \frac{\xi}{2 \eta} \Xi_{0}(\xi, \eta, s) d s d \eta .
\end{aligned}
$$

Таким образом, имеем

$$
\begin{aligned}
T_{\xi}\left[\int_{I_{0}} \int_{0}^{t} \Xi_{0}(\xi, \eta, s) e^{i(2 \eta-\xi)(t-s)} d s d \eta\right] & \\
& =\varepsilon S_{0}+Q_{0}^{+}(\xi) e^{i \xi t}+Q_{0}^{-}(\xi) e^{-i \xi t}+\varepsilon F_{0}(t, \xi),
\end{aligned}
$$

где

$$
\begin{gathered}
Q_{0}^{-}(\xi)=-w_{e} \varepsilon \int_{0}^{\infty} e^{i \xi s} \int_{I_{0}} \frac{\xi}{2 \eta} L_{p \rightarrow s}^{-1}\left(\frac{D(\xi-\eta, \eta)}{p+\frac{1}{\varepsilon}\left[L_{e}-\frac{\xi}{2(\xi-\eta)} u_{e}-\frac{\xi}{2 \eta} w_{e}\right]}\right) d s d \eta \\
Q_{0}^{+}(\xi)=-u_{e} \varepsilon \int_{0}^{\infty} e^{i \xi s} \int_{I_{0}} \frac{\xi}{2(\xi-\eta)} L_{p \rightarrow s}^{-1}\left(\frac{D(\xi-\eta, \eta)}{p+\frac{1}{\varepsilon}\left[L_{e}-\frac{\xi}{2(\xi-\eta)} u_{e}-\frac{\xi}{2 \eta} w_{e}\right]}\right) d s d \eta \\
F_{0}(t, \xi)=\int_{t}^{\infty} \int_{I_{0}}\left(w_{e} \frac{\xi}{2 \eta} e^{-\xi(t-s)}+u_{e} e^{i \xi(t-s)} \frac{\xi}{2(\xi-\eta)}\right) \times \\
\times L_{p \rightarrow s}^{-1}\left(\frac{D(\xi-\eta, \eta)}{p+\frac{1}{\varepsilon}\left[L_{e}-\frac{\xi}{2(\xi-\eta)} u_{e}-\frac{\xi}{2 \eta} w_{e}\right]}\right) d s d \eta
\end{gathered}
$$

1b) Теперь сделаем оценку правых частей в (25). Нетрудно видеть, что

$$
\begin{gathered}
Q_{0}^{-}(\xi)=-w_{e} \varepsilon \int_{0}^{\infty} e^{i \xi s} \int_{I_{0}} \frac{\xi}{2 \eta} L_{p \rightarrow s}^{-1}\left(\frac{D(\xi-\eta, \eta)}{p+\frac{1}{\varepsilon}\left[L_{e}-\frac{\xi}{2(\xi-\eta)} u_{e}-\frac{\xi}{2 \eta} w_{e}\right]}\right) d s d \eta \\
Q_{0}^{+}(\xi)=-u_{e} \int_{0}^{\infty} e^{i \xi s} \int_{I_{0}} \frac{\xi}{2(\xi-\eta)} L_{p \rightarrow s}^{-1}\left(\frac{D(\xi-\eta, \eta)}{p+\frac{1}{\varepsilon}\left[L_{e}-\frac{\xi}{2(\xi-\eta)} u_{e}-\frac{\xi}{2 \eta} w_{e}\right]}\right) d s d \eta .
\end{gathered}
$$

На отрезке $I_{0}$ имеем $\frac{|\xi|}{2|\eta|} \leqslant \frac{1}{2 q}, \frac{|\xi|}{2|\xi-\eta|} \leqslant \frac{1}{2 q}$. Тогда

$$
\begin{aligned}
& \left\|Q_{0}^{-}(\xi)\right\|_{\mathcal{H}^{\sigma}(\mathbb{R})}^{2} \leqslant \frac{1}{2 q^{2}} w_{e}^{2} \varepsilon^{2} \int_{-\infty}^{\infty} \int_{I_{0}}\left|(1+|\eta|)^{\sigma}(1+|\xi-\eta|)^{\sigma} D(\xi-\eta, \eta)\right|^{2} d \eta \times \\
& \quad \times\left\{\int_{I_{0}} \frac{1}{(1+|\eta|)^{2 \sigma}}\left|\int_{0}^{\infty} L_{p \rightarrow s}^{-1}\left(\frac{1}{p+\frac{1}{\varepsilon}\left[L_{e}-\frac{\xi}{2(\xi-\eta)} u_{e}-\frac{\xi}{2 \eta} w_{e}\right]}\right) d s\right|^{2} d \eta+\right. \\
& \left.\quad+\int_{I_{0}} \frac{1}{(1+|\xi-\eta|)^{2 \sigma}}\left|\int_{0}^{\infty} L_{p \rightarrow s}^{-1}\left(\frac{1}{p+\frac{1}{\varepsilon}\left[L_{e}-\frac{\xi}{2(\xi-\eta)} u_{e}-\frac{\xi}{2 \eta} w_{e}\right]}\right) d s\right|^{2} d \eta\right\} d \xi
\end{aligned}
$$


где в силу (24) имеем

$$
\begin{gathered}
\left.\int_{I_{0}} \frac{1}{(1+|\eta|)^{2 \sigma} \mid} \int_{0}^{\infty} L_{p \rightarrow s}^{-1}\left(\frac{1}{p+\frac{1}{\varepsilon}\left[L_{e}-\frac{\xi}{2(\xi-\eta)} u_{e}-\frac{\xi}{2 \eta} w_{e}\right]}\right) d s\right|^{2} d \eta \leqslant \\
\leqslant \frac{2}{a_{0}^{2}} \int_{I_{0}} \frac{d \eta}{(1+|\eta|)^{2 \sigma}}=\frac{2}{a_{0}^{2}} \mu_{\sigma}^{2} .
\end{gathered}
$$

Также оценивается второй член в (26).

2) Теперь исследуем окрестности $I_{2}$, где $|\eta| \geqslant|\xi|-|\xi-\eta| \geqslant(1-q)|\xi|$,

$$
|\xi-2 \eta| \geqslant|\xi|-2|\xi-\eta|>(1-2 q)|\xi|, \quad \text { если } \quad|\xi-\eta|<q|\xi| .
$$

2a) Имеем

$$
\begin{aligned}
\widehat{S_{2}}(p, \xi)=\int_{I_{2}} \frac{D(\xi-\eta, \eta)}{p+i \xi-2 i \eta} d \eta & =\frac{1}{p-i \xi} \int_{I_{2}} D(\xi-\eta, \eta) d \eta- \\
& -2 \int_{I_{2}}(\xi-\eta) D(\xi-\eta, \eta) \frac{1}{(p+i \xi-2 i \eta)(p-i \xi)} d \eta
\end{aligned}
$$

Далее

$$
\begin{aligned}
\left(\frac{i \xi u_{e}}{p-i \xi}-\frac{i \xi w_{e}}{p+i \xi}\right) \frac{1}{p+i \xi-2 i \eta}=-\left(\frac{\xi}{2(\xi-\eta)} u_{e}+\frac{\xi}{2 \eta} w_{e}\right) \frac{1}{p+i \xi-2 i \eta}+ \\
+\frac{\xi}{2(\xi-\eta)} u_{e} \frac{1}{p-i \xi}+\frac{\xi}{2 \eta} w_{e} \frac{1}{p+i \xi}
\end{aligned}
$$

Отсюда

$$
\begin{aligned}
& \left(p+\frac{1}{\varepsilon} L_{e}+\frac{1}{\varepsilon}\left(\frac{i \xi u_{e}}{p-i \xi}-\frac{i \xi w_{e}}{p+i \xi}\right)\right) \int_{I_{2}} \frac{\widehat{\Xi}(\xi, \eta, p)}{p+i \xi-2 i \eta} d \eta= \\
& =\int_{I_{2}}\left(\left[p+\frac{1}{\varepsilon} L_{e}\left(1+\frac{\xi}{2 \eta} \frac{w_{e}}{L_{e}}\right)\right](p-i \xi)+\frac{1}{\varepsilon} i \xi u_{e}\right) \frac{\widehat{\Xi}(\xi, \eta, p)}{(p+i \xi-2 i \eta)(p-i \xi)} d \eta- \\
& \quad-\frac{1}{\varepsilon} w_{e} \frac{1}{p+i \xi} \int_{I_{2}} \frac{\xi}{2 \eta} \widehat{\Xi}(\xi, \eta, p) d \eta
\end{aligned}
$$

где

$$
\begin{aligned}
\mid p^{2}+\left(\frac{1}{\varepsilon} L_{e}(1\right. & \left.\left.+\frac{\xi}{2 \eta} \frac{w_{e}}{L_{e}}\right)-i \xi\right) p- \\
& -i \xi \frac{1}{\varepsilon} L_{e}\left(1+\frac{\xi}{2 \eta} \frac{w_{e}}{L_{e}}-\frac{u_{e}}{L_{e}}\right) \mid \geqslant c_{1}>0, \quad \eta \in I_{2}, \quad \operatorname{Re} p \geqslant-\mu_{0} \varepsilon
\end{aligned}
$$

Доказательство этой оценки аналогично оценке снизу леммы 3.1 в [1]. Здесь

$$
\left[p+\frac{1}{\varepsilon} L_{e}\left(1+\frac{\xi}{2 \eta} \frac{w_{e}}{L_{e}}\right)\right](p-i \xi)+\frac{1}{\varepsilon} i \xi u_{e}=
$$




$$
\begin{aligned}
=(p-i \xi) p & {\left[1+\frac{1}{\varepsilon} L_{e}\left(1+\frac{\xi}{2 \eta} \frac{w_{e}}{L_{e}}\right) \frac{1}{p}+\frac{1}{\varepsilon} u_{e}\left(\frac{1}{(p-i \xi)}-\frac{1}{p}\right)\right]=} \\
= & (p-i \xi) p\left[1+\frac{1}{\varepsilon} L_{e}\left(1+\frac{\xi}{2 \eta} \frac{w_{e}}{L_{e}}-\frac{u_{e}}{L_{e}}\right) \frac{1}{p}+\frac{1}{\varepsilon} u_{e} \frac{1}{(p-i \xi)}\right]
\end{aligned}
$$

где

$\left|1+\frac{1}{\varepsilon} L_{e}\left(1+\frac{\xi}{2 \eta} \frac{w_{e}}{L_{e}}-\frac{u_{e}}{L_{e}}\right) \frac{1}{p}+\frac{1}{\varepsilon} u_{e} \frac{1}{(p-i \xi)}\right| \geqslant c_{1,1}>0, \quad \operatorname{Re} p \geqslant-\mu_{0} \varepsilon, \quad \eta \in I_{2}$.

В то же время для $p=i \xi$ имеем

$$
i \xi+\frac{1}{\varepsilon} L_{e}\left(1+\frac{\xi}{2 \eta} \frac{w_{e}}{L_{e}}\right)-i \xi-\frac{1}{\varepsilon} L_{e}\left(1+\frac{\xi}{2 \eta} \frac{w_{e}}{L_{e}}-\frac{u_{e}}{L_{e}}\right)=\frac{1}{\varepsilon} u_{e} \neq 0 ;
$$

для $p=0$ имеем

$$
i \xi \frac{1}{\varepsilon} L_{e}\left[1+\frac{\xi}{2 \eta} \frac{w_{e}}{L_{e}}-\frac{u_{e}}{L_{e}}\right] \neq 0
$$

если $\xi \neq 0$.

Положим

$$
\widehat{\Xi_{2}}(\xi, \eta, p)=-2 \varepsilon \frac{(\xi-\eta) D(\xi-\eta, \eta)}{\left[p+\frac{1}{\varepsilon} L_{e}\left(1+\frac{\xi}{2 \eta} \frac{w_{e}}{L_{e}}\right)\right](p-i \xi)+\frac{1}{\varepsilon} i \xi u_{e}}, \quad \forall \eta \in I_{2} .
$$

Тогда

$$
\begin{aligned}
& \left(p+\frac{1}{\varepsilon} L_{e}+\frac{1}{\varepsilon}\left(\frac{i \xi u_{e}}{p-i \xi}-\frac{i \xi w u_{e}}{p+i \xi}\right)\right) \int_{I_{2}} \frac{\widehat{\Xi_{2}}(\xi, \eta, p)}{p+i \xi-2 i \eta} d \eta= \\
& \quad=\varepsilon \widehat{S_{2}}(p, \xi)+\varepsilon \frac{1}{p-i \xi} \int_{I_{2}} D(\xi-\eta, \eta) d \eta+w_{e} \frac{1}{p+i \xi} \int_{I_{2}} \frac{\xi}{2 \eta} \widehat{\Xi}(\xi, \eta, p) d \eta .
\end{aligned}
$$

Отсюда

$$
\begin{aligned}
T_{\xi}\left[\int_{I_{2}} \int_{0}^{t} \Xi_{2}(\xi, \eta, s) e^{i(2 \eta-\xi)(t-s)} d s d \eta\right] & = \\
& =\varepsilon S_{2}+Q_{2}^{+}(\xi) e^{i \xi t}+Q_{2}^{-}(\xi) e^{-i \xi t}+F_{2}(t, \xi), \quad \xi \in I_{2},
\end{aligned}
$$

где

$$
\begin{gathered}
Q_{2}^{+}(\xi)=\varepsilon \int_{I_{2}} D(\xi-\eta, \eta) d \eta \\
Q_{2}^{-}(\xi)=-w_{e} \varepsilon \int_{0}^{\infty} e^{i \xi s} \int_{I_{2}} \frac{\xi}{\eta} L_{p \rightarrow s}^{-1}\left(\frac{(\xi-\eta) D(\xi-\eta, \eta)}{\left[p+\frac{1}{\varepsilon} L_{e}\left(1+\frac{\xi}{2 \eta} \frac{w_{e}}{L_{e}}\right)\right](p-i \xi)+\frac{1}{\varepsilon} i \xi u_{e}}\right) d s d \eta, \\
F_{2}(t, \xi)=w_{e} \int_{t}^{\infty} e^{-i \xi(t-s)} \int_{I_{2}} \frac{\xi}{\eta} L_{p \rightarrow s}^{-1}\left(\frac{(\xi-\eta) D(\xi-\eta, \eta)}{\left[p+\frac{1}{\varepsilon} L_{e}\left(1+\frac{\xi}{2 \eta} \frac{w_{e}}{L_{e}}\right)\right](p-i \xi)+\frac{1}{\varepsilon} i \xi u_{e}}\right) d s d \eta .
\end{gathered}
$$


2b) Теперь сделаем оценку правых частей в (29). Нетрудно видеть, что

$$
\left\|Q_{2}^{+}\right\|_{\mathcal{H}^{\sigma}(\mathbb{R})}^{2} \leqslant \varepsilon^{2} \mu_{\sigma}^{2} \int_{-\infty}^{\infty} \int_{I_{2}}(1+|\xi-\eta|)^{2 \sigma}(1+|\eta|)^{2 \sigma}|D(\xi-\eta, \eta)|^{2} d \eta d \xi .
$$

На отрезке $I_{2}$ имеем $|\xi-\eta|<q|\xi|$. Тогда

$$
\begin{aligned}
& \left\|Q_{2}^{-}\right\|_{\mathcal{H}^{\sigma}(\mathbb{R})}^{2} \leqslant \\
& \leqslant \frac{2 w_{e}^{2} \varepsilon^{2} q^{2}}{(1-q)^{2}} \int_{-\infty}^{\infty}\left\{\int_{I_{2}}\left((1+|\xi-\eta|)^{\sigma}(1+|\eta|)^{\sigma}|D(\xi-\eta, \eta)|\right)^{2} d \eta \int_{I_{2}} \frac{1}{(1+|\eta|)^{2 \sigma}} \times\right. \\
& \quad \times\left|\int_{0}^{\infty} L_{p \rightarrow s}^{-1}\left(\frac{|\xi|}{(p-i \xi) p\left[1+\frac{1}{\varepsilon} L_{e}\left(1+\frac{\xi}{2 \eta} \frac{w_{e}}{L_{e}}-\frac{u_{e}}{L_{e}}\right) \frac{1}{p}+\frac{1}{\varepsilon} u_{e} \frac{1}{(p-i \xi)}\right]}\right) d s\right|^{2} d \eta+ \\
& \quad+\int_{I_{2}}\left((1+|\eta|)^{\sigma}(1+|\xi-\eta|)^{\sigma}|D(\xi-\eta, \eta)|\right)^{2} d \eta \int_{I_{2}} \frac{1}{(1+|\xi-\eta|)^{2 \sigma}} \times \\
& \left.\times\left|\int_{0}^{\infty} L_{p \rightarrow s}^{-1}\left(\frac{|\xi|}{(p-i \xi) p\left[1+\frac{1}{\varepsilon} L_{e}\left(1+\frac{\xi}{2 \eta} \frac{w_{e}}{L_{e}}-\frac{u_{e}}{L_{e}}\right) \frac{1}{p}+\frac{1}{\varepsilon} u_{e} \frac{1}{(p-i \xi)}\right]}\right) d s\right|^{2} d \eta\right\} d \xi,
\end{aligned}
$$

где

$$
\begin{aligned}
& \left|\int_{0}^{\infty} L_{p \rightarrow s}^{-1}\left(\frac{|\xi|}{(p-i \xi) p\left[1+\frac{1}{\varepsilon} L_{e}\left(1+\frac{\xi}{2 \eta} \frac{w_{e}}{L_{e}}-\frac{u_{e}}{L_{e}}\right) \frac{1}{p}+\frac{1}{\varepsilon} u_{e} \frac{1}{(p-i \xi)}\right]}\right) d s\right|^{2} \leqslant \\
& \quad \leqslant \frac{1}{2 \gamma_{*}}\left\|L_{p \rightarrow s}^{-1}\left(\frac{|\xi|}{(p-i \xi) p\left[1+\frac{1}{\varepsilon} L_{e}\left(1+\frac{\xi}{2 \eta} \frac{w_{e}}{L_{e}}-\frac{u_{e}}{L_{e}}\right) \frac{1}{p}+\frac{1}{\varepsilon} u_{e} \frac{1}{(p-i \xi)}\right]}\right)\right\|_{L_{2, \gamma_{*}}\left(\mathbb{R}_{+}\right)}^{2}
\end{aligned}
$$

для $\gamma_{*}=\mu_{*} \varepsilon$. Существование такого $\mu_{*} \in(0,1)$ следует из леммы 3.1 в [1], согласно которой ограничены следующие нормы:

$$
\begin{aligned}
& \left\|L_{p \rightarrow s}^{-1}\left(\frac{1+|\xi|}{(p-i \xi) p\left[1+\frac{1}{\varepsilon} L_{e}\left(1+\frac{\xi}{2 \eta} \frac{w_{e}}{L_{e}}-\frac{u_{e}}{L_{e}}\right) \frac{1}{p}+\frac{1}{\varepsilon} u_{e} \frac{1}{(p-i \xi)}\right]}\right)\right\|_{L_{2, \gamma_{*}}\left(\mathbb{R}_{+}\right)}^{2} \leqslant a_{1}^{2}, \\
& \left\|\frac{d}{d t} L_{p \rightarrow s}^{-1}\left(\frac{1}{(p-i \xi) p\left[1+\frac{1}{\varepsilon} L_{e}\left(1+\frac{\xi}{2 \eta} \frac{w_{e}}{L_{e}}-\frac{u_{e}}{L_{e}}\right) \frac{1}{p}+\frac{1}{\varepsilon} u_{e} \frac{1}{(p-i \xi)}\right]}\right)\right\|_{L_{2, \gamma_{*}}\left(\mathbb{R}_{+}\right)}^{2} \leqslant a_{1}^{2} .
\end{aligned}
$$

\section{Отсюда}

$\left\|Q_{2}^{-}\right\|_{\mathcal{H}^{\sigma}(\mathbb{R})}^{2} \leqslant \varepsilon \frac{w_{e}^{2} a_{1}^{2} q^{2} \mu_{\sigma}^{2}}{(1-q)^{2}} \frac{1}{\mu_{*}} \int_{-\infty}^{\infty} \int_{I_{2}}\left((1+|\eta|)^{\sigma}(1+|\xi-\eta|)^{\sigma}|D(\xi-\eta, \eta)|\right)^{2} d \eta d \xi$.

Теперь выберем $\gamma=\mu_{0} \varepsilon, \mu_{0} \in\left(0, \mu_{*}\right)$. Тогда

$$
\begin{gathered}
(1+|\xi|)^{2 \sigma}\left\|F_{2}(t, \xi)\right\|_{L_{2, \gamma}\left(\mathbb{R}_{+}\right)}^{2} \leqslant \frac{2 w_{e}^{2} q^{2}}{(1-q)^{2}} \int_{I_{2}}(1+|\xi-\eta|)^{2 \sigma}(1+|\eta|)^{2 \sigma} \times \\
\times|D(\xi-\eta, \eta)|^{2} d \eta \int_{I_{2}}\left(\frac{1}{(1+|\xi|)^{2 \sigma}}+\frac{1}{(1+|\xi-\eta|)^{2 \sigma}}\right) \times
\end{gathered}
$$


$\times|\xi|^{2}\left\|\int_{t}^{\infty} e^{-i \xi(t-s)} L_{p \rightarrow s}^{-1}\left(\frac{1}{\left[p+\frac{1}{\varepsilon} L_{e}\left(1+\frac{\xi}{2 \eta} \frac{w_{e}}{L_{e}}\right)\right](p-i \xi)+\frac{1}{\varepsilon} i \xi u_{e}}\right) d s\right\|_{L_{2, \gamma}\left(\mathbb{R}_{+}\right)}^{2} d \eta$,

где

$$
\begin{array}{r}
\left\|i \xi \int_{t}^{\infty} e^{-i \xi(t-s)} L_{p \rightarrow s}^{-1}\left(\frac{1}{\left[p+\frac{1}{\varepsilon} L_{e}\left(1+\frac{\xi}{2 \eta} \frac{w_{e}}{L_{e}}\right)\right](p-i \xi)+\frac{1}{\varepsilon} i \xi u_{e}}\right) d s\right\|_{L_{2, \gamma}\left(\mathbb{R}_{+}\right)}^{2} \leqslant \\
\leqslant\left\|\int_{t}^{\infty} e^{-i \xi(t-s)} \frac{d}{d s} L_{p \rightarrow s}^{-1}\left(\frac{1}{\left[p+\frac{1}{\varepsilon} L_{e}\left(1+\frac{\xi}{2 \eta} \frac{w_{e}}{L_{e}}\right)\right](p-i \xi)+\frac{1}{\varepsilon} i \xi u_{e}}\right) d s\right\|_{L_{2, \gamma}\left(\mathbb{R}_{+}\right)}^{2}+ \\
+\left\|L_{p \rightarrow t}^{-1}\left(\frac{1}{\left[p+\frac{1}{\varepsilon} L_{e}\left(1+\frac{\xi}{2 \eta} \frac{w_{e}}{L_{e}}\right)\right](p-i \xi)+\frac{1}{\varepsilon} i \xi u_{e}}\right)\right\|_{L_{2, \gamma}\left(\mathbb{R}_{+}\right)}^{2} .
\end{array}
$$

В силу (28) имеем

$$
\left\|L_{p \rightarrow t}^{-1}\left(\frac{1}{\left[p+\frac{1}{\varepsilon} L_{e}\left(1+\frac{\xi}{2 \eta} \frac{w_{e}}{L_{e}}\right)\right](p-i \xi)+\frac{1}{\varepsilon} i \xi u_{e}}\right)\right\|_{L_{2, \gamma}\left(\mathbb{R}_{+}\right)}^{2} \leqslant a_{1}^{2} .
$$

В то же время

$$
\begin{aligned}
& \left|\int_{t}^{\infty} e^{-i \xi(t-s)} \frac{d}{d s} L_{p \rightarrow s}^{-1}\left(\frac{1}{\left[p+\frac{1}{\varepsilon} L_{e}\left(1+\frac{\xi}{2 \eta} \frac{w_{e}}{L_{e}}\right)\right](p-i \xi)+\frac{1}{\varepsilon} i \xi u_{e}}\right) d s\right|^{2} \leqslant \\
& \quad \leqslant \frac{1}{2 \gamma_{*}} e^{-2 \gamma_{*} t}\left\|\frac{d}{d s} L_{p \rightarrow s}^{-1}\left(\frac{1}{\left[p+\frac{1}{\varepsilon} L_{e}\left(1+\frac{\xi}{2 \eta} \frac{w_{e}}{L_{e}}\right)\right](p-i \xi)+\frac{1}{\varepsilon} i \xi u_{e}}\right)\right\|_{L_{2, \gamma_{*}\left(\mathbb{R}_{+}\right)}^{2}}
\end{aligned}
$$

\section{Отсюда}

$$
\begin{gathered}
\left\|\int_{t}^{\infty} e^{-i \xi(t-s)} \frac{d}{d s} L_{p \rightarrow s}^{-1}\left(\frac{1}{\left[p+\frac{1}{\varepsilon} L_{e}\left(1+\frac{\xi}{2 \eta} \frac{w_{e}}{L_{e}}\right)\right](p-i \xi)+\frac{1}{\varepsilon} i \xi u_{e}}\right) d s\right\|_{L_{2, \gamma}\left(\mathbb{R}_{+}\right)}^{2} \leqslant \\
\quad \leqslant \frac{1}{4 \gamma_{*}\left(\gamma_{*}-\gamma\right)}\left\|\frac{1}{d s} L_{p \rightarrow s}^{-1}\left(\frac{1}{\left[p+\frac{1}{\varepsilon} L_{e}\left(1+\frac{\xi}{2 \eta} \frac{w_{e}}{L_{e}}\right)\right](p-i \xi)+\frac{1}{\varepsilon} i \xi u_{e}}\right)\right\|_{L_{2, \gamma_{*}\left(\mathbb{R}_{+}\right)}}^{2}
\end{gathered}
$$

Следовательно

$$
\varepsilon^{2}\left\|F_{2}(t, \xi)\right\|_{L_{2, \gamma}\left(\mathbb{R}_{+} ; \mathcal{H}^{\sigma}(\mathbb{R})\right)}^{2} \leqslant \frac{w_{e}^{2} q^{2} \mu_{\sigma}^{2}}{(1-q)^{2} \mu_{*}\left(\mu_{*}-\mu_{0}\right)}\|D\|_{\mathcal{H}^{\sigma, \sigma}\left(\mathbb{R}^{2}\right)}^{2} .
$$

3) Теперь осталось исследовать окрестности $I_{1}$, где $|\xi-\eta| \geqslant|\xi|-|\eta| \geqslant$ $\geqslant(1-q)|\xi|$

$$
|\xi-2 \eta| \geqslant|\xi|-2|\eta|>(1-2 q)|\xi|, \quad \text { если }|\eta|<q|\xi|,
$$




$$
\begin{aligned}
\widehat{S_{1}}(p, \xi)=\int_{I_{1}} \frac{D(\xi-\eta, \eta)}{p-i \xi-2 i(\eta-\xi)} d \eta=\frac{1}{p+i \xi} \int_{I_{1}} D(\xi-\eta, \eta) d \eta- \\
\quad-2 \int_{I_{1}} \frac{\eta D(\xi-\eta, \eta)}{(p+i \xi-2 i \eta)(p+i \xi)} d \eta
\end{aligned}
$$

Также получим

$$
\begin{aligned}
\left(p+\frac{1}{\varepsilon} L_{e}+\frac{1}{\varepsilon}\left(\frac{i \xi u_{e}}{p-i \xi}-\frac{i \xi w_{e}}{p+i \xi}\right)\right) \int_{I_{1}} \frac{\widehat{\Xi_{1}}(\xi, \eta, p)}{p+i \xi-2 i \eta} d \eta= & \\
=\int_{I_{1}}\left(\left[p+\frac{1}{\varepsilon} L_{e}\left(1-\frac{u_{e}}{L_{e}} \frac{\xi}{2(\xi-\eta)}\right)\right]\right. & \left.-i \xi \frac{1}{\varepsilon} w_{e}\right) \frac{\widehat{\Xi_{1}}(\xi, \eta, p)}{(p+i \xi-2 i \eta)(p+i \xi)} d \eta+ \\
& +\frac{1}{\varepsilon} u_{e} \frac{1}{p-i \xi} \int_{I_{2}} \frac{\xi}{2(\xi-\eta)} \widehat{\Xi_{1}}(\xi, \eta, p) d \eta .
\end{aligned}
$$

В этом случае доказательство, аналогичное приведённому в лемме 3.1 [1], позволяет получить оценку

$$
\left|\left[p+\frac{1}{\varepsilon} L_{e}\left(1-\frac{u_{e}}{L_{e}} \frac{\xi}{2(\xi-\eta)}\right)\right](p+i \xi)-i \frac{1}{\varepsilon} \xi w_{e}\right| \geqslant c_{2}>0, \eta \in I_{1}, \operatorname{Re} p \geqslant-\mu_{0} \varepsilon .
$$

Здесь

$$
\begin{aligned}
& {\left[p+\frac{1}{\varepsilon} L_{e}\left(1-\frac{u_{e}}{L_{e}} \frac{\xi}{2(\xi-\eta)}\right)\right](p+i \xi)-i \frac{1}{\varepsilon} \xi w_{e}=} \\
& \quad=p(p+i \xi)\left(1+\frac{1}{\varepsilon} L_{e}\left(1-\frac{u_{e}}{L_{e}} \frac{\xi}{2(\xi-\eta)}-\frac{w_{e}}{L_{e}}\right) \frac{1}{p}+\frac{1}{\varepsilon} w_{e} \frac{1}{(p+i \xi)}\right)
\end{aligned}
$$

где

$$
\left|1+\frac{1}{\varepsilon} L_{e}\left(1-\frac{u_{e}}{L_{e}} \frac{\xi}{2(\xi-\eta)}-\frac{w_{e}}{L_{e}}\right) \frac{1}{p}+\frac{1}{\varepsilon} w_{e} \frac{1}{(p+i \xi)}\right| \geqslant c_{2,1}>0,
$$

$\eta \in I_{1}, \quad \operatorname{Re} p \geqslant-\mu_{0} \varepsilon$.

Заметим, что

а) при $p=-\xi$

$$
\left.\left(\left[p+\frac{1}{\varepsilon} L_{e}\left(1-\frac{u_{e}}{L_{e}} \frac{\xi}{2(\xi-\eta)}\right)\right](p+i \xi)-i \frac{1}{\varepsilon} \xi w_{e}\right)\right|_{p=-\xi}=-i \frac{1}{\varepsilon} \xi w_{e} \neq 0
$$

b) при $p=0$

$$
\begin{aligned}
\left(\left[p+\frac{1}{\varepsilon} L_{e}\left(1-\frac{u_{e}}{L_{e}} \frac{\xi}{2(\xi-\eta)}\right)\right](p\right. & \left.+i \xi)-i \frac{1}{\varepsilon} \xi w_{e}\right)\left.\right|_{p=0}= \\
& =i \xi \frac{1}{\varepsilon} L_{e}\left(1-\frac{u_{e}}{L_{e}} \frac{\xi}{2(\xi-\eta)}-\frac{w_{e}}{L_{e}}\right) \neq 0
\end{aligned}
$$

если $\xi \neq 0$. 


\section{Положим}

$$
\widehat{\Xi_{1}}(\xi, \eta, p)=\frac{4 \varepsilon \eta D(\xi-\eta, \eta)}{\left[p+\frac{1}{\varepsilon} L_{e}\left(1-\frac{u_{e}}{L_{e}} \frac{\xi}{2(\xi-\eta)}\right)\right](p+i \xi)-i \frac{1}{\varepsilon} \xi w_{e}}, \quad \eta \in I_{1} .
$$

Тогда

$$
\begin{aligned}
& T_{\xi}\left[\int_{I_{1}} \int_{0}^{t} \Xi_{1}(\xi, \eta, s) e^{i(2 \eta-\xi)(t-s)} d s d \eta\right]= \\
&=\varepsilon S_{1}+Q_{1}^{+}(\xi) e^{i \xi t}+Q_{1}^{-}(\xi) e^{-i \xi t}+\varepsilon F_{1}(t, \xi), \quad \xi \in I_{1},
\end{aligned}
$$

где

$$
Q_{1}^{-}(\xi)=\varepsilon \int_{I_{1}} e^{i \xi s} D(\xi-\eta, \eta) d \eta
$$

$$
\begin{aligned}
& Q_{1}^{+}(\xi)=-2 u_{e} \varepsilon \int_{0}^{\infty} e^{-i \xi s} \int_{I_{1}} \frac{\xi}{\xi-\eta} L_{p \rightarrow s}^{-1} \times \\
& \times\left(\frac{\eta D(\xi-\eta, \eta)}{\left[p+\frac{1}{\varepsilon} L_{e}\left(1-\frac{u_{e}}{L_{e}} \frac{\xi}{2(\xi-\eta)}\right)\right](p+i \xi)-i \frac{1}{\varepsilon} \xi w_{e}}\right) d s d \eta \\
& F_{1}(t, \xi)=2 u_{e} \int_{t}^{\infty} e^{i \xi(t-s)} \int_{I_{1}} \frac{\xi}{\xi-\eta} \times \\
& \quad \times L_{p \rightarrow s}^{-1}\left(\frac{\eta D(\xi-\eta, \eta)}{\left[p+\frac{1}{\varepsilon} L_{e}\left(1-\frac{u_{e}}{L_{e}} \frac{\xi}{2(\xi-\eta)}\right)\right](p+i \xi)-i \frac{1}{\varepsilon} \xi w_{e}}\right) d s d \eta .
\end{aligned}
$$

Так же как выше, получим

$$
\begin{gathered}
\left\|Q_{1}^{-}\right\|_{\mathcal{H}^{\sigma}(\mathbb{R})}^{2} \leqslant \varepsilon^{2} \mu_{\sigma}^{2} \int_{-\infty}^{\infty} \int_{I_{1}}(1+|\xi-\eta|)^{2 \sigma}(1+|\eta|)^{2 \sigma}|D(\xi-\eta, \eta)|^{2} d \eta d \xi \\
\left\|Q_{1}^{+}\right\|_{\mathcal{H}^{\sigma}(\mathbb{R})}^{2} \leqslant \\
\leqslant \varepsilon \frac{2 w_{e}^{2} a_{1}^{2} q^{2} \mu_{\sigma}^{2}}{(1-q)^{2}} \frac{\varepsilon}{\gamma} \int_{-\infty}^{\infty} \int_{I_{1}}\left((1+|\eta|)^{\sigma}(1+|\xi-\eta|)^{\sigma}|D(\xi-\eta, \eta)|\right)^{2} d \eta d \xi \\
\varepsilon^{2}\left\|F_{1}(t, \xi)\right\|_{L_{2, \gamma}\left(\mathbb{R}_{+} ; \mathcal{H}^{\sigma}(\mathbb{R})\right)}^{2} \leqslant \frac{w_{e}^{2} q^{2} \mu_{\sigma}^{2}}{(1-q)^{2} \mu_{*}\left(\mu_{*}-\mu_{0}\right)}\|D\|_{\mathcal{H}^{\sigma, \sigma}\left(\mathbb{R}^{2}\right)}^{2} .
\end{gathered}
$$

Здесь, так же как выше, мы воспользовались существованием $\mu_{*} \in(0,1)$ в силу лемы 3.1 [1], поскольку для $\gamma_{*}=\mu_{*} \varepsilon$ ограничены нормы:

$$
\begin{aligned}
& \left\|L_{p \rightarrow t}^{-1}\left(\frac{1+|\xi|}{p(p+i \xi)\left(1+\frac{1}{\varepsilon} L_{e}\left(1-\frac{u_{e}}{L_{e}} \frac{\xi}{2(\xi-\eta)}-\frac{w_{e}}{L_{e}}\right) \frac{1}{p}+\frac{1}{\varepsilon} w_{e} \frac{1}{(p+i \xi)}\right)}\right)\right\|_{L_{2, \gamma_{*}}\left(\mathbb{R}_{+}\right)}^{2} \leqslant a_{1}^{2}, \\
& \left\|\frac{d}{d t} L_{p \rightarrow t}^{-1}\left(\frac{1}{p(p+i \xi)\left(1+\frac{1}{\varepsilon} L_{e}\left(1-\frac{u_{e}}{L_{e}} \frac{\xi}{2(\xi-\eta)}-\frac{w_{e}}{L_{e}}\right) \frac{1}{p}+\frac{1}{\varepsilon} w_{e} \frac{1}{(p+i \xi)}\right)}\right)\right\|_{L_{2, \gamma_{*}}\left(\mathbb{R}_{+}\right)}^{2} \leqslant a_{1}^{2} .
\end{aligned}
$$


4) Итак, в разных областях

$$
\begin{aligned}
& \widehat{\Xi}(\xi, \eta, p)= \begin{cases}-4 \varepsilon \frac{\eta D(\xi-\eta, \eta)}{\left(p+\frac{1}{\varepsilon} L_{e}\left(1-\frac{u_{e}}{L_{e}} \frac{\xi}{2(\xi-\eta)}\right)\right)(p+i \xi)-i \frac{1}{\varepsilon} \xi w_{e}}, & \eta \in I_{1}, \\
-2 \varepsilon \frac{(\xi-\eta) D(\xi-\eta, \eta)}{\left(p+\frac{1}{\varepsilon} L_{e}\left(1+\frac{\xi}{2 \eta} \frac{w_{e}}{L_{e}}\right)\right)(p-i \xi)+\frac{1}{\varepsilon} i \xi u_{e}}, & \eta \in I_{2}, \\
\varepsilon \frac{D(\xi-\eta, \eta)}{p+\frac{1}{\varepsilon}\left(L_{e}-\frac{\xi}{2(\xi-\eta)} u_{e}-\frac{\xi}{2 \eta} w_{e}\right)}, & \eta \in I_{0} ;\end{cases} \\
& Q^{+}(\xi)= \begin{cases}-2 u_{e} \varepsilon \int_{0}^{\infty} e^{-i \xi s} \int_{I_{1}} \frac{\xi}{\xi-\eta} \times & \\
\times L_{p \rightarrow s}^{-1}\left(\frac{\eta D(\xi-\eta, \eta)}{\left[p+\frac{1}{\varepsilon} L_{e}\left(1-\frac{u_{e}}{L_{e}} \frac{\xi}{2(\xi-\eta)}\right)\right](p+i \xi)-i \frac{1}{\varepsilon} \xi w_{e}}\right) d s d \eta, & \eta \in I_{1}, \\
\varepsilon \int_{I_{2}} D(\xi-\eta, \eta) d \eta, & \eta \in I_{2}, \\
-u_{e} \varepsilon \int_{0}^{\infty} e^{i \xi s} \int_{I_{0}} \frac{\xi}{2(\xi-\eta)} \times L_{p \rightarrow s}^{-1}\left(\frac{1 \xi-\eta, \eta)}{p+\frac{1}{\varepsilon}\left[L_{e}-\frac{\xi}{2(\xi-\eta)} u_{e}-\frac{\xi}{2 \eta} w_{e}\right]}\right) d s d \eta, & \eta \in I_{0} ;\end{cases}
\end{aligned}
$$

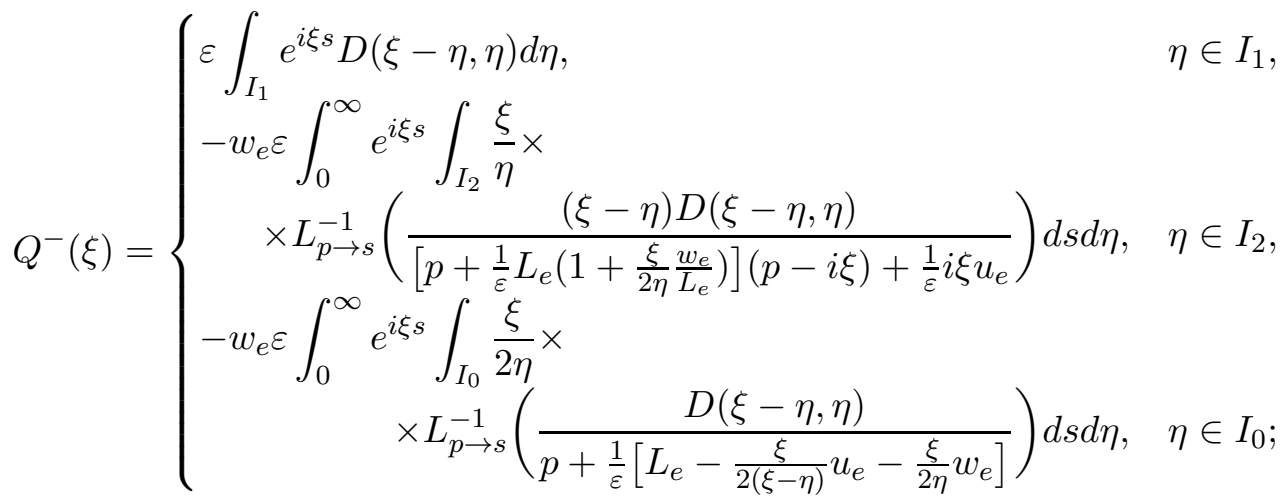

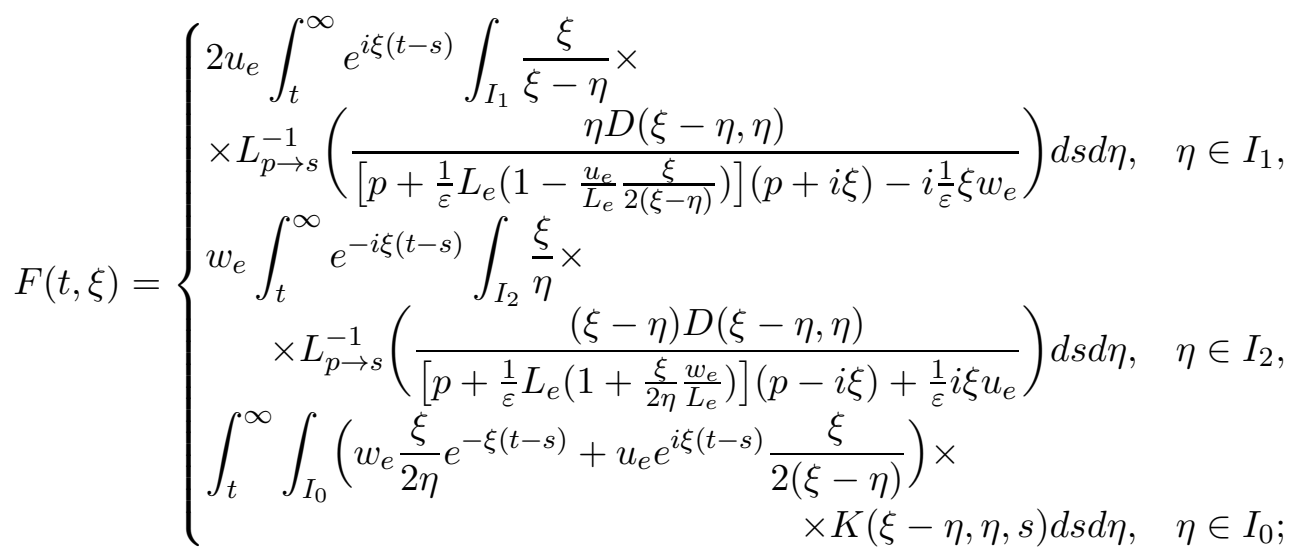




$$
K(\xi-\eta, \eta, s)=L_{p \rightarrow s}^{-1}\left(\frac{D(\xi-\eta, \eta)}{p+\frac{1}{\varepsilon}\left[L_{e}-\frac{\xi}{2(\xi-\eta)} u_{e}-\frac{\xi}{2 \eta} w_{e}\right]}\right)
$$

Структура решения.

Положим

$$
Z_{j}(t, \xi)=\int_{I_{j}} \int_{0}^{t} e^{i(2 \eta-\xi)(t-s)} \Xi(\xi, \eta, s) d s d \eta, \quad j=0,1,2 .
$$

Тогда $Z(t, \xi)=Z_{0}(t, \xi)+Z_{1}(t, \xi)+Z_{2}(t, \xi)$ - решение задачи Коши (23).

а) Здесь

$$
\begin{array}{r}
Z_{0}(t, \xi)=\varepsilon \int_{I_{0}} D(\xi-\eta, \eta) \int_{0}^{t} e^{i(2 \eta-\xi)(t-s)} e^{-\frac{1}{\varepsilon}\left(L_{e}-\frac{\xi}{2(\xi-\eta)} u_{e}-\frac{\xi}{2 \eta} w_{e}\right) s} d s d \eta= \\
=\varepsilon \int_{I_{0}} e^{i(2 \eta-\xi) t} \Psi_{0}(\xi-\eta, \eta) d \eta+\varepsilon G_{0}(t, \xi)
\end{array}
$$

где

$$
\begin{gathered}
\Psi_{0}(\xi-\eta, \eta)=\frac{D(\xi-\eta, \eta)}{i(2 \eta-\xi)+\frac{1}{\varepsilon}\left(L_{e}-\frac{\xi}{2(\xi-\eta)} u_{e}-\frac{\xi}{2 \eta} w_{e}\right)}, \\
G_{0}(t, \xi)=\int_{I_{0}} e^{-\frac{1}{\varepsilon}\left(L_{e}-\frac{\xi}{2(\xi-\eta)} u_{e}-\frac{\xi}{2 \eta} w_{e}\right) t} \frac{D(\xi-\eta, \eta)}{-i(2 \eta-\xi)-\frac{1}{\varepsilon}\left(L_{e}-\frac{\xi}{2(\xi-\eta)} u_{e}-\frac{\xi}{2 \eta} w_{e}\right)} d \eta .
\end{gathered}
$$

b) Далее преобразование Лапласа $Z_{1}(t, \xi)$ по $t$ :

$$
\begin{aligned}
& \widehat{Z_{1}}(p, \xi)=\varepsilon \widehat{J_{1}}+\varepsilon \widehat{J}_{2}, \\
& \widehat{J_{1}}=\int_{I_{1}} \frac{D(\xi-\eta, \eta)}{p\left(1+\frac{1}{\varepsilon} L_{e}\left(1-\frac{u_{e}}{L_{e}} \frac{\xi}{2(\xi-\eta)}-\frac{w_{e}}{L_{e}}\right) \frac{1}{p}+\frac{1}{\varepsilon} w_{e} \frac{1}{(p+i \xi)}\right)} \frac{1}{(p+i \xi-2 i \eta)} d \eta, \\
& \widehat{J_{2}}=-\frac{1}{(p+i \xi)} \int_{I_{1}} \frac{D(\xi-\eta, \eta)}{p\left(1+\frac{1}{\varepsilon} L_{e}\left(1-\frac{u_{e}}{L_{e}} \frac{\xi}{2(\xi-\eta)}-\frac{w_{e}}{L_{e}}\right) \frac{1}{p}+\frac{1}{\varepsilon} w_{e} \frac{1}{(p+i \xi)}\right)} d \eta .
\end{aligned}
$$

\section{Положим}

$$
\widehat{M}_{1}(\xi-\eta, \eta, p)=\frac{D(\xi-\eta, \eta)}{p\left(1+\frac{1}{\varepsilon} L_{e}\left(1-\frac{u_{e}}{L_{e}} \frac{\xi}{2(\xi-\eta)}-\frac{w_{e}}{L_{e}}\right) \frac{1}{p}+\frac{1}{\varepsilon} w_{e} \frac{1}{(p+i \xi)}\right)} .
$$

Тогда

$$
\begin{aligned}
& J_{2}(t, \xi)=\int_{0}^{t} e^{-i \xi(t-s)} \int_{I_{1}} M_{1}(\xi-\eta, \eta, s) d \eta d s= \\
&=e^{-i \xi t} \int_{0}^{\infty} e^{i \xi s} \int_{I_{1}} M_{1}(\xi-\eta, \eta, s) d \eta d s+
\end{aligned}
$$




$$
+\int_{t}^{\infty} e^{-i \xi(t-s)} \int_{I_{1}} M_{1}(\xi-\eta, \eta, s) d \eta d s
$$

- обратное преобразование Лапласа $J_{2}$ второго члена $\widehat{J}_{2}$ в правой части $(30)$, где $M_{1}(\xi-\eta, \eta, s)=L_{p \rightarrow s}^{-1}\left(\widehat{M}_{1}(\xi-\eta, \eta, p)\right)$.

Вопрос в том, что есть $\widehat{J_{1}}$ ? Обратное преобразование Лапласа $J_{1}$ первого члена в правой части (30):

$$
\begin{aligned}
J_{1}(t, \xi)=\int_{I_{1}} \int_{0}^{t} e^{-i(\xi-2 \eta)(t-s)} M_{1}(\xi-\eta, \eta, s) d s d \eta= \\
=\int_{I_{1}} e^{-i(\xi-2 \eta) t} \int_{0}^{\infty} e^{i(\xi-2 \eta) s} M_{1}(\xi-\eta, \eta, s) d s d \eta- \\
\quad-\int_{I_{1}} \int_{t}^{\infty} e^{-i(\xi-2 \eta)(t-s)} M_{1}(\xi-\eta, \eta, s) d s d \eta
\end{aligned}
$$

Отсюда

$$
Z_{1}(t, \xi)=\varepsilon \int_{I_{1}} e^{-i(\xi-2 \eta) t} \Psi_{1}(\xi-\eta, \eta) d \eta+R_{1}^{-}(\xi) e^{-i \xi t}+\varepsilon G_{1}(t, \xi)
$$

где

$$
\begin{gathered}
\Psi_{1}(\xi-\eta, \eta)=\int_{0}^{\infty} M_{1}(\xi-\eta, \eta, s) d s, R_{1}^{-}(\xi)=\varepsilon \int_{0}^{\infty} e^{i \xi s} \int_{I_{1}} M_{1}(\xi-\eta, \eta, s) d s d \eta \\
G_{1}(t, \xi)=-\int_{t}^{\infty} e^{-i \xi(t-s)} \int_{I_{1}} M_{1}(\xi-\eta, \eta, s) d \eta d s+ \\
+\int_{I_{1}} \int_{t}^{\infty} e^{-i(\xi-2 \eta)(t-s)} M_{1}(\xi-\eta, \eta, s) d s d \eta
\end{gathered}
$$

Проведём оценку $\Psi_{1}, R_{1}^{-}, G_{1}$. Имеем

$$
\begin{aligned}
\int_{-\infty}^{\infty}(1+|\xi|)^{2 \sigma}\left|\int_{I_{1}} e^{-i(\xi-2 \eta) t} \Psi_{1}(\xi-\eta, \eta) d \eta\right|^{2} d \xi \leqslant & \\
\leqslant & 2 \int_{-\infty}^{\infty}\left|\int_{I_{1}}(1+|\xi-\eta|)^{\sigma} e^{-i(\xi-2 \eta) t} \Psi_{1}(\xi-\eta, \eta) d \eta\right|^{2} d \xi+ \\
& +2 \int_{-\infty}^{\infty}\left|\int_{I_{1}}(1+|\eta|)^{\sigma} e^{-i(\xi-2 \eta) t} \Psi_{1}(\xi-\eta, \eta) d \eta\right|^{2} d \xi \leqslant \\
& \leqslant 4 \mu_{\sigma}^{2}\left\|\Psi_{1}\right\|_{\mathcal{H}^{\sigma, \sigma}\left(\mathbb{R}^{2}\right)}^{2}
\end{aligned}
$$

где $\Psi_{1}=0$ вне $I_{1}$. Здесь 


$$
\begin{aligned}
& \int_{-\infty}^{\infty}\left|\int_{I_{1}}(1+|\xi-\eta|)^{\sigma} e^{-i(\xi-2 \eta) t} \Psi_{1}(\xi-\eta, \eta) d \eta\right|^{2} d \xi \leqslant \\
& \leqslant \int_{-\infty}^{\infty} \mid \int_{I_{1}}(1+|\xi-\eta|)^{\sigma} e^{-i(\xi-2 \eta) t} \times \\
& \quad \times\left.\int_{0}^{\infty} L_{p \rightarrow s}^{-1} \frac{D(\xi-\eta, \eta)}{p\left(1+\frac{1}{\varepsilon} L_{e}\left(1-\frac{u_{e}}{L_{e}} \frac{\xi+\eta}{2 \xi}-\frac{w_{e}}{L_{e}}\right) \frac{1}{p}+\frac{1}{\varepsilon} w_{e} \frac{1}{(p+i(\xi+\eta))}\right)} d s d \eta\right|^{2} d \xi \leqslant \\
& \quad \leqslant \frac{1}{\varepsilon} \frac{a_{1}^{2} \mu_{\sigma}^{2}}{2 \mu_{0}}\|D\|_{\mathcal{H}^{\sigma, \sigma}\left(\mathbb{R}^{2}\right)}^{2}
\end{aligned}
$$

Также получим, что

$$
\int_{-\infty}^{\infty}\left|\int_{I_{1}}(1+|\eta|)^{\sigma} e^{-i(\xi-2 \eta) t} \Psi_{1}(\xi-\eta, \eta) d \eta\right|^{2} d \xi \leqslant \frac{1}{\varepsilon} \frac{a_{1}^{2} \mu_{\sigma}^{2}}{2 \mu_{0}}\|D\|_{\mathcal{H}^{\sigma, \sigma}\left(\mathbb{R}^{2}\right)}^{2}
$$

где

$$
\max _{\xi \in \mathbb{R}, \eta \in I_{1}}\left\|L_{p \rightarrow t}^{-1} \frac{1}{p\left(1+\frac{1}{\varepsilon} L_{e}\left(1-\frac{u_{e}}{L_{e}} \frac{\xi}{2(\xi-\eta)}-\frac{w_{e}}{L_{e}}\right) \frac{1}{p}+\frac{1}{\varepsilon} w_{e} \frac{1}{(p+i \xi)}\right)}\right\|_{L_{2, \gamma}(\mathbb{R})} \leqslant a_{1} .
$$

Отсюда

$$
\begin{gathered}
\varepsilon\left\|\int_{I_{1}} e^{-i(\xi-2 \eta) t} \Psi_{1}(\xi-\eta, \eta) d \eta\right\|_{W_{\infty}^{1}\left(\mathbb{R}_{+} ; \mathcal{H}^{\sigma}(\mathbb{R})\right)} \\
\leqslant \varepsilon^{1 / 2} \frac{a_{1} \mu_{\sigma}}{\mu_{0}^{1 / 2}}\left(\|D\|_{\mathcal{H}^{\sigma+1, \sigma}\left(\mathbb{R}^{2}\right)}+\|D\|_{\mathcal{H}^{\sigma, \sigma+1}\left(\mathbb{R}^{2}\right)}\right),
\end{gathered}
$$

где

$$
\|g\|_{W_{\infty}^{1}\left(\mathbb{R}_{+} ; \mathcal{H}^{\sigma}(\mathbb{R})\right)}=\left\|\frac{d}{d t} g\right\|_{L_{\infty}\left(\mathbb{R}_{+} ; \mathcal{H}^{\sigma}(\mathbb{R})\right)}+\|g\|_{L_{\infty}\left(\mathbb{R}_{+} ; \mathcal{H}^{\sigma}(\mathbb{R})\right)}
$$

Также

$$
\left\|e^{-i \xi t} R_{1}^{-}(\xi)\right\|_{W_{\infty}^{1}\left(\mathbb{R}_{+} ; \mathcal{H}^{\sigma}(\mathbb{R})\right)}^{2} \leqslant \varepsilon^{1 / 2} \frac{a_{1} \mu_{\sigma}}{\mu_{0}^{1 / 2}}\left(\|D\|_{\mathcal{H}^{\sigma+1, \sigma}\left(\mathbb{R}^{2}\right)}+\|D\|_{\mathcal{H}^{\sigma, \sigma+1}\left(\mathbb{R}^{2}\right)}\right) .
$$

\section{При этом}

$$
\begin{aligned}
\varepsilon^{2}\left\|G_{1}\right\|_{W_{2, \gamma}^{1}\left(\mathbb{R}_{+} ; \mathcal{H}^{\sigma}(\mathbb{R})\right)}^{2} \leqslant \frac{2 a_{1}^{2} \mu_{\sigma}^{2}}{\mu_{*}\left(\mu_{*}-\mu_{0}\right)} \int_{-\infty}^{\infty} \int_{I_{1}}(1+|\eta|)^{2 \sigma}(1+|\xi-\eta|)^{2 \sigma} \times \\
\quad \times\left((1+|\xi-\eta|)^{2}+(1+|\eta|)^{2 \sigma}\right)|D(\xi-\eta, \eta)|^{2} d \eta d \xi .
\end{aligned}
$$

с) В то же время

$$
\widehat{Z_{2}}(p, \xi)=\varepsilon \widehat{J}_{1}+\varepsilon \widehat{J}_{2}
$$


$\widehat{J}_{1}=-\int_{I_{2}} \widehat{M}_{2}(\xi-\eta, \eta, p) \frac{1}{(p+i \xi-2 i \eta)} d \eta, \widehat{J}_{2}=\frac{1}{(p-i \xi)} \int_{I_{2}} \widehat{M}_{2}(\xi-\eta, \eta, p) d \eta$,

где

$$
\widehat{M}_{2}(\xi-\eta, \eta, p)=\frac{D(\xi-\eta, \eta)}{p\left[1+\frac{1}{\varepsilon} L_{e}\left(1+\frac{\xi}{2 \eta} \frac{w_{e}}{L_{e}}-\frac{u_{e}}{L_{e}}\right) \frac{1}{p}+\frac{1}{\varepsilon} u_{e} \frac{1}{(p-i \xi)}\right]}
$$

Тогда

$$
\begin{aligned}
J_{2}(t, \xi)=e^{i \xi t} \int_{0}^{\infty} e^{-i \xi s} \int_{I_{2}} M_{2}(\xi-\eta, \eta, s) d \eta d s+ & \\
& +\int_{t}^{\infty} e^{i \xi(t-s)} \int_{I_{2}} M_{2}(\xi-\eta, \eta, s) d \eta d s
\end{aligned}
$$

- обратное преобразование Лапласа $J_{2}$ второго члена $\widehat{J}_{2}$ в правой части $(31)$, где $M_{2}(\xi-\eta, \eta, s)=L_{p \rightarrow s}^{-1}\left(\widehat{M}_{2}(\xi-\eta, \eta, p)\right)$. Обратное преобразование Лапласа $J_{1}$ первого члена $\widehat{J}_{1}$ правой части $(31)$ :

$$
\begin{aligned}
& J_{1}(t, \xi)=-\int_{I_{2}} \int_{0}^{t} e^{-i(\xi-2 \eta)(t-s)} M_{2}(\xi-\eta, \eta, s) d s d \eta= \\
& =-\int_{I_{2}} e^{-i(\xi-2 \eta) t} \int_{0}^{\infty} e^{i(\xi-2 \eta) s} M_{2}(\xi-\eta, \eta, s) d s d \eta+ \\
& \quad+\int_{I_{2}} \int_{t}^{\infty} e^{-i(\xi-2 \eta)(t-s)} M_{2}(\xi-\eta, \eta, s) d s d \eta .
\end{aligned}
$$

Отсюда

$$
Z_{2}(t, \xi)=\int_{I_{2}} e^{-i(\xi-2 \eta) t} \Psi_{2}(\xi-\eta, \eta) d \eta+R_{2}^{+}(\xi) e^{i \xi t}+\varepsilon G_{2}(t, \xi),
$$

где

$$
\begin{gathered}
\Psi_{2}(\xi-\eta, \eta)=-\int_{0}^{\infty} e^{i(\xi-2 \eta) s} \mathcal{M}_{2}(\xi-\eta, \eta, s) d s \\
R_{2}^{+}(\xi)=\varepsilon \int_{0}^{\infty} e^{-i \xi s} \int_{I_{2}} M_{2}(\xi-\eta, \eta, s) d \eta d s \\
G_{2}(t, \xi)=\int_{t}^{\infty} e^{i \xi(t-s)} \int_{I_{2}} M_{2}(\xi-\eta, \eta, s) d \eta d s+ \\
\quad+\int_{I_{2}} \int_{t}^{\infty} e^{-i(\xi-2 \eta)(t-s)} M_{2}(\xi-\eta, \eta, s) d s d \eta
\end{gathered}
$$

Так же как выше, получим оценки

$$
\begin{aligned}
\varepsilon\left\|\int_{I_{2}} e^{-i(\xi-2 \eta) t} \Psi_{2}(\xi-\eta, \eta) d \eta\right\|_{W_{\infty}^{1}\left(\mathbb{R}_{+} ; \mathcal{H}^{\sigma}(\mathbb{R})\right)} & \leqslant \\
& \leqslant \varepsilon^{1 / 2} \frac{a_{2} \mu_{\sigma}}{\mu_{0}^{1 / 2}}\left(\|D\|_{\mathcal{H}^{\sigma+1, \sigma}\left(\mathbb{R}^{2}\right)}+\|D\|_{\mathcal{H}^{\sigma, \sigma+1}\left(\mathbb{R}^{2}\right)}\right),
\end{aligned}
$$




$$
\begin{gathered}
\left\|e^{-i \xi t} R_{2}^{+}(\xi)\right\|_{W_{\infty}^{1}\left(\mathbb{R}_{+} ; \mathcal{H}^{\sigma}(\mathbb{R})\right)}^{2} \leqslant \varepsilon^{1 / 2} \frac{a_{2} \mu_{\sigma}}{\mu_{0}^{1 / 2}}\left(\|D\|_{\mathcal{H}^{\sigma+1, \sigma}\left(\mathbb{R}^{2}\right)}+\|D\|_{\mathcal{H}^{\sigma, \sigma+1}\left(\mathbb{R}^{2}\right)}\right) \\
\varepsilon^{2}\left\|G_{2}\right\|_{W_{2, \gamma}^{1}\left(\mathbb{R}_{+} ; \mathcal{H}^{\sigma}(\mathbb{R})\right)}^{2} \leqslant \frac{2 a_{2}^{2} \mu_{\sigma}^{2}}{\mu_{*}\left(\mu_{*}-\mu_{0}\right)} \int_{-\infty}^{\infty} \int_{I_{2}}(1+|\eta|)^{2 \sigma}(1+|\xi-\eta|)^{2 \sigma} \times \\
\times\left((1+|\xi-\eta|)^{2}+(1+|\eta|)^{2 \sigma}\right)|D(\xi-\eta, \eta)|^{2} d \eta d \xi .
\end{gathered}
$$

d) И последнее. Суммируя, получим структуру решения

$$
\begin{aligned}
Z(t, \xi)= & Z_{0}(t, \xi)+Z_{1}(t, \xi)+Z_{2}(t, \xi)= \\
& =\varepsilon \int_{-\infty}^{\infty} e^{-i(\xi-2 \eta) t} \Psi(\xi-\eta, \eta) d \eta+R_{2}^{+}(\xi) e^{i \xi t}+R_{1}^{-}(\xi) e^{-i \xi t}+G(t, \xi)
\end{aligned}
$$

где

$$
\begin{gathered}
G(t, \xi)=G_{0}(t, \xi)+G_{1}(t, \xi)+G_{2}(t, \xi), \\
\Psi(\xi, \eta)= \begin{cases}\Psi_{0}(\xi, \eta), & \eta \in I_{0}, \\
\Psi_{1}(\xi, \eta), & \eta \in I_{1}, \\
\Psi_{2}(\xi, \eta), & \eta \in I_{2} .\end{cases}
\end{gathered}
$$

Сделав обратное преобразование Фурье $\xi \rightarrow x$, получим

$$
\mathcal{Z}_{D}(t, x)=\varepsilon N_{D}(x-t, x+t)+\mathcal{R}_{D}^{-}(x-t)+\mathcal{R}_{D}^{+}(x+t)+\mathcal{G}_{D}(x, t),
$$

где $Z_{D}(t, \xi), R_{D}^{ \pm}, G_{D}(x, \xi)$ - преобразование Фурье $\mathcal{Z}_{D}(t, x), \mathcal{R}_{d}^{ \pm}(t, x)$ и $\mathcal{G}_{D}(x, t)$ по $x$ соответственно. Лемма 6 доказана.

В дальнейшем решение $Z(t, \xi)$ задачи Коши $(22)$ с точностью до правой части $Q^{+}(\xi) e^{i \xi t}+Q^{-}(\xi) e^{-i \xi t}+F(t, \xi)$ для

$$
S=S_{D}=\int_{-\infty}^{\infty} e^{-i(\xi-2 \eta) t} D(\xi-\eta, \eta) d \eta
$$

будем обозначать через

$$
Z(t, \xi)=T_{\xi}^{-1}\left(\int_{-\infty}^{\infty} e^{-i(\xi-2 \eta) t} D(\xi-\eta, \eta) d \eta\right)
$$

Соответственно $\mathcal{Z}(t, x)$ - обратное преобразование Фурье:

$$
Z(t, \xi)=T_{\xi}^{-1}\left(\int_{-\infty}^{\infty} e^{-i(\xi-2 \eta) t} D(\xi-\eta, \eta) d \eta\right) \quad(\xi \rightarrow x)
$$

12. Билинейные формы. Теперь мы должны перенести результаты леммы 6 на задачу Коши для нелинейного уравнения

$$
T_{\xi} y(t, \xi)=2 \varepsilon v_{e}^{1 / 2}(B(y, y)+L(y))-2 \varepsilon \int_{-\infty}^{\infty} D^{-}(\xi-\eta) D^{+}(\eta) e^{-i(\xi-2 \eta) t} d \eta
$$


с точностью до правой части $R^{+}(\xi) e^{i \xi t}+R^{-}(\xi) e^{-i \xi t}+G(t, \xi)$. Рассмотрим вклад билинейных форм в случае

$$
\begin{aligned}
y(t, \xi)=\int_{0}^{t} \int_{-\infty}^{\infty} e^{-(\xi-2 \eta)(t-s)} \Xi_{D}(\xi-\eta, \eta, s) d s d \eta+ & \\
& +T_{\xi}^{-1}\left(\Omega_{\Upsilon}(t, \xi)\right)+T_{\xi}^{-1}(Y(t, \xi)), \quad Y(t, \xi) \in L_{2, \gamma}\left(\mathbb{R}_{+}, \mathcal{H}^{\sigma}\right),
\end{aligned}
$$

где $\Omega_{\Upsilon}=\Upsilon_{-}(\xi) e^{-i \xi t}+\Upsilon_{+}(\xi) e^{i \xi t}$. Тогда

$$
\begin{aligned}
& B(y, y)=B\left(y_{D}, y_{D}\right)+L\left(y_{D}\right)+B\left(y_{D}, T_{\xi}^{-1}\left(\Omega_{\Upsilon}\right)\right)+B\left(T_{\xi}^{-1}\left(\Omega_{\Upsilon}\right), y_{D}\right)+ \\
& +B\left(T_{\xi}^{-1}\left(\Omega_{\Upsilon}\right), T_{\xi}^{-1}\left(\Omega_{\Upsilon}\right)+L\left(T_{\xi}^{-1}\left(\Omega_{\Upsilon}\right)\right)+B\left(y_{D}+T_{\xi}^{-1}\left(\Omega_{\Upsilon}\right), T^{-1} Y\right)+\right. \\
& +B\left(T^{-1} Y, y_{D}+T_{\xi}^{-1}\left(\Omega_{\Upsilon}\right)+B\left(T^{-1} Y, T^{-1} Y\right)+L\left(T^{-1} Y\right)\right.
\end{aligned}
$$

Случай $y=y_{D}$.

Сначала исследуем вклад билинейных форм в случае, когда

$$
\begin{aligned}
y(t, \xi)= & y_{D}(t, \xi)=\int_{0}^{t} \int_{-\infty}^{\infty} e^{-i(\xi-2 \eta)(t-s)} \Xi_{D}(\xi-\eta, \eta, s) d s d \eta= \\
& =\int_{-\infty}^{\infty} e^{-i(\xi-2 \eta) t} \Psi_{D}(\xi-\eta, \eta) d \eta+R_{D}^{+}(\xi) e^{i \xi t}+R_{D}^{-}(\xi) e^{-i \xi t}+G_{D}(t, \xi)
\end{aligned}
$$

и $\Xi_{D}, \Psi_{D}, R_{D}^{ \pm}, G_{D}$ определяются леммой предыдущего параграфа. Тогда для обратного преобразование Фурье по $x$

$$
F_{\xi \rightarrow x}^{-1}(y)(t, x)=\varepsilon N_{D}(x-t, x+t)+\mathcal{R}_{D}^{-}(x-t)+\mathcal{R}_{D}^{+}(x+t)+\mathcal{G}_{D}(x, t),
$$

здесь билинейная форма

$$
\begin{aligned}
B(y, y)= & \int_{-\infty}^{\infty}\{y(t, \xi-\eta) y(t, \eta)- \\
& -\frac{1}{4}\left(y(t, \xi-\eta)-i(\xi-\eta) \int_{0}^{t} e^{-i(\xi-\eta)(t-s)} y(s, \xi-\eta) d s\right) \times \\
& \left.\times\left(y(t, \eta)+i \eta \int_{0}^{t} e^{i \eta(t-s)} y(s, \eta) d s\right)\right\} d \eta \\
y(t, \xi-\eta)-i(\xi-\eta) \int_{0}^{t} e^{-i(\xi-\eta)(t-s)} y(s, \xi-\eta) d s & =\int_{0}^{t} e^{-i(\xi-\eta)(t-s)} \frac{d}{d s} y(s, \xi-\eta) d s \\
y(t, \eta)+i \eta \int_{0}^{t} e^{i \eta(t-s)} y(s, \eta) d s & =\int_{0}^{t} e^{i \eta(t-s)} \frac{d}{d s} y(s, \eta) d s .
\end{aligned}
$$


Лемма 7. Пусть $\Xi_{D}, \Psi_{D}, R_{D}^{ \pm}, G_{D}$ определяются леммой 6 . Тогда существуют функиии $D_{B}(\xi, \eta), Q_{B}^{ \pm}(\xi), F_{B}(t, \xi)$ такие, что

$$
B(y, y)=\varepsilon \int_{-\infty}^{\infty} D_{B}(\xi-\eta, \eta) e^{-i(\xi-2 \eta) t} d \eta+Q_{B}^{-}(\xi) e^{-i \xi t}+Q_{B}^{+}(\xi) e^{i \xi t}+\varepsilon F_{B}(t, \xi)
$$

для которых справедливы оценки

$$
\begin{gathered}
\varepsilon\left\|\int_{-\infty}^{\infty} D_{B}(\xi-\eta, \eta) e^{-i(\xi-2 \eta) t} d \eta\right\|_{L_{\infty}\left(\mathbb{R}_{+} ; \mathcal{H}^{\sigma}(\mathbb{R})\right)} \leqslant \\
\leqslant c_{1} \varepsilon^{1 / 2}\left(\|D\|_{\mathcal{H}^{\sigma+1, \sigma}\left(\mathbb{R}^{2}\right)}+\|D\|_{\mathcal{H}^{\sigma, \sigma+1}\left(\mathbb{R}^{2}\right)}\right) \\
\varepsilon\left\|Q \pm_{B}(\xi) e^{-i \xi t}\right\|_{L_{\infty}\left(\mathbb{R}_{+} ; \mathcal{H}^{\sigma}(\mathbb{R})\right)} \leqslant c_{1} \varepsilon^{1 / 2}\left(\|D\|_{\mathcal{H}^{\sigma+1, \sigma}\left(\mathbb{R}^{2}\right)}+\|D\|_{\mathcal{H}^{\sigma, \sigma+1}\left(\mathbb{R}^{2}\right)}\right) \\
\varepsilon\left\|F_{B}\right\|_{L_{2, \gamma}\left(\mathbb{R}_{+} ; \mathcal{H}^{\sigma}(\mathbb{R})\right)} \leqslant c_{1}\left(\|D\|_{\mathcal{H}^{\sigma+1, \sigma}\left(\mathbb{R}^{2}\right)}+\|D\|_{\mathcal{H}^{\sigma, \sigma+1}\left(\mathbb{R}^{2}\right)}\right)
\end{gathered}
$$

Доказательств о леммы 7 проводится аналогично приведённым выше. Оценки построенного решения следуют из оценок леммы 6.

Случай $y=y_{D}$ для формъ $L(y)$.

Теперь исследуем вклад билинейной формы

$$
\begin{aligned}
L(y)=\int_{-\infty}^{\infty}\left\{\frac { 1 } { 2 } \frac { 1 } { v _ { e } ^ { 1 / 2 } } \left(D^{-}(\xi-\eta) e^{-i(\xi-\eta) t} \int_{0}^{t} e^{i \eta(t-s)} \frac{d}{d s} y(s, \eta) d s+\right.\right. \\
\left.+D^{+}(\eta) e^{i \eta t} \int_{0}^{t} e^{-i(\xi-\eta)(t-s)} \frac{d}{d s} y(s, \xi-\eta) d s\right)+ \\
\quad+e^{-\frac{1}{\varepsilon} L_{e} t}\left[\widetilde{v}^{0}(\xi-\eta) y(t, \eta)+y(t, \xi-\eta) \widetilde{v}^{0}(\eta)-\right. \\
-\frac{1}{4} v^{0}(\xi-\eta) \frac{\frac{1}{\varepsilon} L_{e}}{\frac{1}{\varepsilon} L_{e}-i(\xi-\eta)} \int_{0}^{t} e^{i \eta(t-s)} \frac{d}{d s} y(s, \eta) d s- \\
\left.\left.\quad-\frac{1}{4} v^{0}(\eta) \frac{\frac{1}{\varepsilon} L_{e}}{\frac{1}{\varepsilon} L_{e}+i \eta} \int_{0}^{t} e^{-i(\xi-\eta)(t-s)} \frac{d}{d s} y(s, \xi-\eta) d s\right]\right\} d \eta
\end{aligned}
$$

Лемма 8. Пусть выполнены условия лемм 6 и 7. Тогда существуют функu, ии $D_{L}(\xi, \eta), Q_{L}^{ \pm}(\xi), F_{L}(t, \xi)$ maкuе, что

$$
L(y)=\varepsilon \int_{-\infty}^{\infty} D_{L}(\xi-\eta, \eta) e^{-i(\xi-2 \eta) t} d \eta+Q_{L}^{-}(\xi) e^{-i \xi t}+Q_{L}^{+}(\xi) e^{i \xi t}+\varepsilon F_{L} B(t, \xi)
$$

для которых справедливы оценки

$$
\begin{gathered}
\varepsilon\left\|\int_{-\infty}^{\infty} D_{L}(\xi-\eta, \eta) e^{-i(\xi-2 \eta) t} d \eta\right\|_{L_{\infty}\left(\mathbb{R}_{+} ; \mathcal{H}^{\sigma}(\mathbb{R})\right)} \\
\leqslant c_{1} \varepsilon^{1 / 2}\left(\|D\|_{\mathcal{H}^{\sigma+1, \sigma}\left(\mathbb{R}^{2}\right)}+\|D\|_{\mathcal{H}^{\sigma, \sigma+1}\left(\mathbb{R}^{2}\right)}\right) \\
\end{gathered}
$$




$$
\begin{aligned}
& \varepsilon\left\|Q \pm_{L}(\xi) e^{-i \xi t}\right\|_{L_{\infty}\left(\mathbb{R}_{+} ; \mathcal{H}^{\sigma}(\mathbb{R})\right)} \leqslant c_{1} \varepsilon^{1 / 2}\left(\|D\|_{\mathcal{H}^{\sigma+1, \sigma}\left(\mathbb{R}^{2}\right)}+\|D\|_{\mathcal{H}^{\sigma, \sigma+1}\left(\mathbb{R}^{2}\right)}\right) \\
& \varepsilon\left\|F_{L}\right\|_{L_{2, \gamma}\left(\mathbb{R}_{+} ; \mathcal{H}^{\sigma}(\mathbb{R})\right)} \leqslant c_{1}\left(\|D\|_{\mathcal{H}^{\sigma+1, \sigma}\left(\mathbb{R}^{2}\right)}+\|D\|_{\mathcal{H}^{\sigma, \sigma+1}\left(\mathbb{R}^{2}\right)}\right) .
\end{aligned}
$$

Доказ а тель ст в о леммы 7 проводится аналогично приведённым выше.

Случай для борм $B\left(y_{D}, T_{\xi}^{-1}\left(\Omega_{\Upsilon}\right)\right)+B\left(T_{\xi}^{-1}\left(\Omega_{\Upsilon}\right), y_{D}\right)+B\left(T_{\xi}^{-1}\left(\Omega_{\Upsilon}\right), T_{\xi}^{-1}\left(\Omega_{\Upsilon}\right)\right)+$ $+L\left(T_{\xi}^{-1}\left(\Omega_{\Upsilon}\right)\right)$.

Теперь рассмотрим вклад билинейных форм

$$
B\left(y_{D}, T_{\xi}^{-1}\left(\Omega_{\Upsilon}\right)\right)+B\left(T_{\xi}^{-1}\left(\Omega_{\Upsilon}\right), y_{D}\right)+B\left(T_{\xi}^{-1}\left(\Omega_{\Upsilon}\right), T_{\xi}^{-1}\left(\Omega_{\Upsilon}\right)\right)+L\left(T_{\xi}^{-1}\left(\Omega_{\Upsilon}\right)\right)
$$

где

$$
\left.\Omega_{\Upsilon}\right)(t, \xi)=\Upsilon^{+}(\xi) e^{i \xi t}+\Upsilon^{-}(\xi) e^{-i \xi t}
$$

Лемма 9. Пусть выполнены условия леммы 6. Тогда существуют $D_{\Omega}(\xi, \eta)$, $\Upsilon_{\Omega}^{ \pm}(\xi), F_{\Omega}(t, \xi)$ maкuе, чmо

$$
\begin{gathered}
B\left(y_{D}, T_{\xi}^{-1}\left(\Omega_{\Upsilon}\right)\right)+B\left(T_{\xi}^{-1}\left(\Omega_{\Upsilon}\right), y_{D}\right)+B\left(T_{\xi}^{-1}\left(\Omega_{\Upsilon}\right), T_{\xi}^{-1}\left(\Omega_{\Upsilon}\right)\right)+L\left(T_{\xi}^{-1}\left(\Omega_{\Upsilon}\right)\right)= \\
=\int_{-\infty}^{\infty} e^{-(\xi-2 \eta) t} D_{\Omega}(\xi-\eta, \eta) d \eta+Q_{\Omega}^{-}(\xi) e^{-i \xi t}+Q_{\Omega}^{+}(\xi) e^{i \xi t}+F_{\Omega}(t, \xi)
\end{gathered}
$$

и справедливы оценки

$$
\begin{gathered}
\varepsilon\left\|\int_{-\infty}^{\infty} D_{\Omega}(\xi-\eta, \eta) e^{-i(\xi-2 \eta) t} d \eta\right\|_{L_{\infty}\left(\mathbb{R}_{+} ; \mathcal{H}^{\sigma}(\mathbb{R})\right)} \leqslant \\
\leqslant c_{2} \varepsilon^{1 / 2}\left(\|D\|_{\mathcal{H}^{\sigma+1, \sigma}\left(\mathbb{R}^{2}\right)}+\|D\|_{\mathcal{H}^{\sigma, \sigma+1}\left(\mathbb{R}^{2}\right)}\right) \\
\varepsilon\left\|Q_{\Omega}^{ \pm}(\xi) e^{-i \xi t}\right\|_{L_{\infty}\left(\mathbb{R}_{+} ; \mathcal{H}^{\sigma}(\mathbb{R})\right)} \leqslant c_{2} \varepsilon^{1 / 2}\left(\|D\|_{\mathcal{H}^{\sigma+1, \sigma}\left(\mathbb{R}^{2}\right)}+\|D\|_{\mathcal{H}^{\sigma, \sigma+1}\left(\mathbb{R}^{2}\right)}\right) \\
\varepsilon\left\|F_{\Omega}\right\|_{L_{2, \gamma}\left(\mathbb{R}_{+} ; \mathcal{H}^{\sigma}(\mathbb{R})\right)} \leqslant c_{2}\left(\|D\|_{\mathcal{H}^{\sigma+1, \sigma}\left(\mathbb{R}^{2}\right)}+\|D\|_{\mathcal{H}^{\sigma, \sigma+1}\left(\mathbb{R}^{2}\right)}\right) .
\end{gathered}
$$

Доказательство леммы 8 проводится аналогично приведённым выше.

\section{3. Сведение к нелинейному уравнению в гильбертовом пространстве} $\boldsymbol{L}_{\mathbf{2}, \boldsymbol{\gamma}}\left(\mathbb{R}_{+} ; \mathcal{H}^{\boldsymbol{\sigma}}\right)$. Наша задача свести уравнение

$$
\begin{aligned}
& T_{\xi} y(t, \xi)=\frac{1}{\varepsilon} h(t, \xi)+H(t, \xi)+2 \varepsilon v_{e}^{1 / 2} B(y, y)+2 \varepsilon v_{e}^{1 / 2} L(y)+ \\
&+2 \frac{1}{\varepsilon}\left(\frac{w_{e}}{v_{e}^{1 / 2}} D^{-}(\xi) e^{-i \xi t}+\frac{u_{e}}{v_{e}^{1 / 2}} D^{+}(\xi) e^{i \xi t}\right)- \\
&-2 \varepsilon \int_{-\infty}^{\infty} D^{-}(\xi-\eta) D^{+}(\eta) e^{-i(\xi-2 \eta) t} d \eta
\end{aligned}
$$

к нелинейному уравнению в гильбертовом пространстве $L_{2, \gamma}\left(\mathbb{R}_{+} ; \mathcal{H}^{\sigma}\right)$. 
Решение (33) будем искать в виде

$$
\begin{aligned}
& y(t, \xi)=\int_{0}^{t} \int_{-\infty}^{\infty} e^{-(\xi-2 \eta)(t-s)} \Xi_{D}(\xi-\eta, \eta, s) d s d \eta+ \\
& \quad+T_{\xi}^{-1}\left(\Upsilon_{-}(\xi) e^{-i \xi t}+\Upsilon_{+}(\xi) e^{i \xi t}\right)+T_{\xi}^{-1}(Y(t, \xi)), \quad Y(t, \xi) \in L_{2, \gamma}\left(R_{+}, \mathcal{H}^{\sigma}\right) .
\end{aligned}
$$

Тогда в силу лемм 6-8 (33) примет следующий вид:

$$
\begin{aligned}
& T_{\xi} y_{D}(t, \xi)+ \Omega_{\Upsilon}(t, \xi)+Y(t, \xi)=\frac{1}{\varepsilon} h(t, \xi)+H(t, \xi)+ \\
&+2 \varepsilon \int_{-\infty}^{\infty} e^{-i(\xi-2 \eta) t}\left(v_{e}^{1 / 2} \varepsilon\left(D_{B}+D_{L}+D_{\Omega}\right)(\xi-\eta, \eta)-D^{-}(\xi-\eta) D^{+}(\eta)\right) d \eta+ \\
&+2 \frac{1}{\varepsilon}\left(\left[\frac{w_{e}}{v_{e}^{1 / 2}} D^{-}(\xi)+\varepsilon^{2} v_{e}^{1 / 2}\left(Q_{B}^{-}+Q_{L}^{-}+Q_{\Omega}^{-}\right)(\xi)\right] e^{-i \xi t}+\right. \\
&\left.+\left[\frac{u_{e}}{v_{e}^{1 / 2}} D^{+}(\xi)+\varepsilon^{2} v_{e}^{1 / 2}\left(Q_{B}^{+}+Q_{L}^{+}+Q_{\Omega}^{+}\right)(\xi)\right] e^{i \xi t}\right)+ \\
&+2 \varepsilon^{2} v_{e}^{1 / 2}\left(F_{\Omega}(t, \xi)+F_{Y}(t, \xi)\right)+2 \varepsilon v_{e}^{1 / 2}\left(B\left(T^{-1} Y, T^{-1} Y\right)+L\left(T^{-1} Y\right)\right)
\end{aligned}
$$

где

$$
\begin{gathered}
\left.y_{D}(t, \xi)\right)=\int_{0}^{t} \int_{-\infty}^{\infty} e^{-i(\xi-2 \eta)(t-s)} \Xi_{D}(\xi-\eta, \eta, s) d \eta d s, \\
\Omega_{\Upsilon}(t, \xi)=\Upsilon^{+}(\xi) e^{i \xi t}+\Upsilon^{-}(\xi) e^{-i \xi t}
\end{gathered}
$$

В правой части (34) мы получили секулярные (неинтегрируемые члены). Чтобы их уничтожить, достаточно в силу первой части леммы 6 выбрать $D(\xi, \eta)$ в $\Xi_{D}(\xi-\eta, \eta, s)$ из уравнения

$$
D(\xi, \eta)=2\left(v_{e}^{1 / 2} \varepsilon\left(D_{B}+D_{L}+D_{\Omega}\right)(\xi, \eta)-D^{-}(\xi) D^{+}(\eta)\right)
$$

и функции $\Upsilon^{ \pm}(\xi)$ из уравнений

$$
\begin{aligned}
& \Upsilon^{-}(\xi)=2 \frac{1}{\varepsilon}\left(\frac{w_{e}}{v_{e}^{1 / 2}} D^{-}(\xi)+\varepsilon^{2} v_{e}^{1 / 2}\left(Q_{B}^{-}+Q_{L}^{-}+Q_{\Omega}^{-}\right)(\xi)\right) \\
& \Upsilon^{+}(\xi)=2 \frac{1}{\varepsilon}\left(\frac{u_{e}}{v_{e}^{1 / 2}} D^{+}(\xi)+\varepsilon^{2} v_{e}^{1 / 2}\left(Q_{B}^{+}+Q_{L}^{+}+Q_{\Omega}^{+}\right)(\xi)\right)
\end{aligned}
$$

Тогда мы сведём уравнение (33) к нелинейному уравнению в гильбертовом пространстве $L_{2, \gamma}\left(\mathbb{R}_{+} ; \mathcal{H}^{\sigma}\right)$ :

$$
\begin{aligned}
Y(t, \xi)=\frac{1}{\varepsilon} h(t, \xi)+H(t, \xi)+2 \varepsilon^{2} & v_{e}^{1 / 2}\left(F_{B}(t, \xi)+F_{\Omega}(t, \xi)+F_{Y}(t, \xi)\right)+ \\
& +2 \varepsilon v_{e}^{1 / 2}\left(B\left(T^{-1} Y, T^{-1} Y\right)+L\left(T^{-1} Y\right)\right)
\end{aligned}
$$

существование решения которого следует из результатов теоремы 1. 
14. Условие несекулярности (общий случай). Таким образом, у нас возникли секулярные неинтегрируемые члены, которые мы можем аннулировать, решив систему алгебраических уравнений

$$
\begin{aligned}
& D(\xi, \eta)=-2 D^{-}(\xi) D^{+}(\eta)+2 v_{e}^{1 / 2} \varepsilon\left(D_{B}+D_{L}+D_{\Omega}\right)(\xi, \eta), \\
& \Upsilon^{-}(\xi)=\frac{1}{\varepsilon} \frac{2 w_{e}}{v_{e}^{1 / 2}} D^{-}(\xi)+2 \varepsilon v_{e}^{1 / 2}\left(Q_{B}^{-}(\xi)+Q_{L}^{-}(\xi)+Q_{\Omega}^{-}(\xi)\right), \\
& \Upsilon^{+}(\xi)=\frac{1}{\varepsilon} \frac{2 u_{e}}{v_{e}^{1 / 2}} D^{+}(\xi)+2 \varepsilon v_{e}^{1 / 2}\left(Q_{B}^{+}(\xi)+Q_{L}^{+}(\xi)+Q_{\Omega}^{+}(\xi)\right),
\end{aligned}
$$

которую мы назовём условием несекулярности.

Доказательство существования решения $D(\xi, \eta), \Upsilon^{ \pm}(\xi)$ условия несекулярности аналогично доказательству разрешимости условия несекулярности в [1]. И, как мы отмечали выше, разрешимость условия несекулярности позволяет свести уравнение (34) к уравнению (35) в гильбертовом пространстве $L_{2, \gamma}\left(\mathbb{R}_{+} ; \mathcal{H}^{\sigma}\right)$, существование решения которого следует из результатов теоремы 1. Это заканчивает исследование общего случая комплекснозначных начальных данных.

Замечание. Приведённые выше рассуждения имеют общий характер, что позволяет перенести доказательства на двумерное и трёхмерное кинетические уравнения, естественно, с большими техническими сложностями, что не является предметом печатаемой статьи. Более того, свойства дискретных кинетических уравнений близки к свойствам кинетического уравнения Больцмана-Пайерса [7], что даёт надежду на доказательство подобных результатов для кинетического уравнения Больцмана-Пайерса.

Работа выполнена при поддержке РФФИ (гранты №№ 09-01-12024, 09-01-00288, 11-01-12082-офи_м).

\section{БИБЛИОГРАФИЧЕСКИЙ СПИСОК}

1. Е. В. Радкевич, "О существовании глобальных решений задачи Коши для дискретных кинетических уравнений (непериодический случай)" // Пробл. мат. анал., 2012. Т. 62; англ. пер.: E. V. Radkevich, "The existence of global solutions to the cauchy problem for discrete kinetic equations" // J. Math. Sci., New York, 2012. Vol. 181, no. 2. Pp. 232-280.

2. T. E. Broadwell, "Study of rarified shear flow by the discrete velocity method" // J. Fluid Mech., 1964. Vol. 19, no. 3. Pp. 401-414.

3. С. К. Годунов, У. М. Султангазин, "О дискретных моделях кинетического уравнения Больцмана" // УМH, 1971. Т. 26, №3(159). С. 3-51; англ. пер.: S. K. Godunov, U. M. Sultangazin, "On discrete models of the kinetic Boltzmann equation" // Russian Math. Surveys, 1971. Vol. 26, no. 3. Pp. 1-56.

4. L. Boltzmann, "On the Maxwell method to the reduction of hydrodynamic equations from the kinetic gas theory" / Rep. Brit. Assoc. London, 1894. Рp. 579; русск. пер.: Л. Больцман, "О максвелловском методе вывода уравнений гидродинамики из кинетической теории газов" / В сб.: Избранные труды: Молекулярно-кинетическая теория газов. Термодинамика. Статистическая механика. Теория излучения. Общие вопросы физики; ред. Л. С. Полак. М.: Наука, 1984. С. 307-308.

5. В. В. Веденяпин, Кинетические уравнения Больцмана и Власова. М.: Физматлит, 2001. 112 c. [V. V. Vedenyapin, Kinetic Boltzmann and Vlasov Equations. Moscow: Fizmatlit, 2001. 112 pp.]

6. S. Chapman, T. G. Cowling, The mathematical theory of non-uniform gases. An account 
of the kinetic theory of viscosity, thermal conduction and diffusion in gases. Cambridge: Cambridge University Press, 1970. xxiv+423 pp.

7. R. Peierls, "Zur kinetischen Theorie der Wärmeleitung in Kristallen" // Ann. Phys., 1929. Vol. 395, no. 8. Pp. 1055-1101.

Поступила в редакцию $18 / \mathrm{X} / 2012$;

в окончательном варианте - 25/XII/2012.

MSC: 35Q20; 35C20, 35Q82, 82B40

\section{ON PROBLEM OF NONEXISTENCE OF DISSIPATIVE ESTIMATE FOR DISCRETE KINETIC EQUATIONS}

\section{E. V. Radkevich}

M. V. Lomonosov Moscow State University, Faculty of Mechanics and Mathematics, Vorob'evy gory, Moscow, Russia, 119899.

E-mail: evrad07@gmail.com

The existence of a global solution to the discrete kinetic equations in Sobolev spaces is proved, its decomposition by summability is obtained, the influence of its oscillations generated by the interaction operator is explored. The existence of a submanifold $\mathcal{M}_{\text {diss }}$ of initial data $\left(u^{0}, v^{0}, w^{0}\right)$ for which the dissipative solution exists is proved. It's shown that the interaction operator generates the solitons (progressive waves) as the nondissipative part of the solution when the initial data $\left(u^{0}, v^{0}, w^{0}\right)$ deviate from the submanifold $\mathcal{M}_{\text {diss }}$. The amplitude of solitons is proportional to the distance from $\left(u^{0}, v^{0}, w^{0}\right)$ to the submanifold $\mathcal{M}_{\text {diss. }}$. It follows that the solution can stabilize as $t \rightarrow \infty$ only on compact sets of spatial variables.

Key words: dissipative estimates, discrete kinetic equations.

Original article submitted 18/X/2012;

revision submitted 25/XII/2012.

Evgenii V. Radkevich (Dr. Sci. (Phys. \& Math.)), Professor, Dept. of Differential Equations. 